\title{
A STUDY OF $n$-Lie-ISOCLINIC LEIBNIZ ALGEBRAS
}

\author{
G. R. Biyogmam ${ }^{1}$ and J. M. Casas ${ }^{2}$ \\ ${ }^{1}$ Department of Mathematics, Georgia College \& State University \\ Campus Box 17 Milledgeville, GA 31061-0490 \\ E-mail address: guy.biyogmam@gcsu.edu \\ ${ }^{2}$ Dpto. Matemática Aplicada, Universidad de Vigo, E. E. Forestal \\ Campus Universitario A Xunqueira, 36005 Pontevedra, Spain \\ E-mail address: jmcasas@uvigo.es
}

\begin{abstract}
In this paper we introduce the concept of $n$-Lie-isoclinism on nonLie Leibniz algebras. Among the results obtained, we provide several characterizations of $n$-Lie-isoclinic classes of Leibniz algebras. Also, we provide a characterization of $n$-Lie-stem Leibniz algebras, and prove that every $n$-Lie-isoclinic class of Leibniz algebras contains a $n$-Lie-stem Leibniz algebra.
\end{abstract}

2010 MSC: 17A32, 17B30.

Key words: $n$-Lie isoclinism; $n$-Lie-stem Leibniz algebras, $n$-Lie-central extension, $n$-Lie center.

\section{Introduction}

The concept of isoclinism was introduced in 1939 by P. Hall in his attempt to classify $p$-groups using an equivalence relation weaker than the notion of isomorphism [10. Later himself generalized the notion of isoclinism to that of isologism in [11, which is in fact isoclinism with respect to a certain variety of groups. When the variety of all the trivial groups is considered, then the notion of isomorphism is recovered. When the chosen variety is the variety of all abelian groups, then the notion of isoclinism is recovered. When the variety of all nilpotent groups of class at most $n$ is considered, then arises the notion of $n$-isoclinism.

This concept has been widely studied in several algebraic structures. For example, it is worth mentioning [2, 9, 13, 17, 20] in case of groups; [12] in case of pairs of groups; in case of Lie algebras [18, 19] and [16] in the case of pairs of Lie algebras.

Recently, the concept of isoclinism of Lie algebras has been considered in the relative context, given rise to the notion of Lie-isoclinism of Leibniz algebras [3]. Relative means that the notion of Lie-isoclinism arises through the Liezation functor $(-)_{\text {Lie }}:$ Leib $\rightarrow$ Lie which assigns the Lie algebra $\mathfrak{g}_{\text {Lie }}=\mathfrak{g} /<\{[x, x]$ : 
$x \in \mathfrak{g}\}>$ to a given Leibniz algebra $\mathfrak{g}$, while the classical notion of isoclinism arises through the abelianization functor, which assigns to a Leibniz algebra $\mathfrak{g}$ the abelian Leibniz algebra $\frac{\mathfrak{g}}{[\mathfrak{g}, \mathfrak{g}]}$. This philosophy comes from the categorical theory of central extensions relative to a chosen Birkhoff subcategory of a semiabelian category. We refer to [3, 7, 8] and references given therein for a detailed explanation.

Our goal in this article is to study the notion of $n$-isoclinism relative to the Liezation functor, that is we consider the variety of Lie-nilpotent non-Lie Leibniz algebras of class at most $n$, called as $n$-Lie-isoclinism, which is an equivalence relation between two Leibniz algebras $\mathfrak{g}_{1}$ and $\mathfrak{g}_{2}$ for which there exist two isomorphisms $\eta: \frac{\mathfrak{g}_{1}}{\mathcal{Z}_{n}^{\text {Lie }}\left(\mathfrak{g}_{1}\right)} \rightarrow \frac{\mathfrak{g}_{2}}{\mathcal{Z}_{n}^{\text {Lie }}\left(\mathfrak{g}_{2}\right)}$ and $\xi: \gamma_{n+1}^{\text {Lie }}\left(\mathfrak{g}_{1}\right) \rightarrow \gamma_{n+1}^{\text {Lie }}\left(\mathfrak{g}_{2}\right)$ such that the following diagram is commutative:

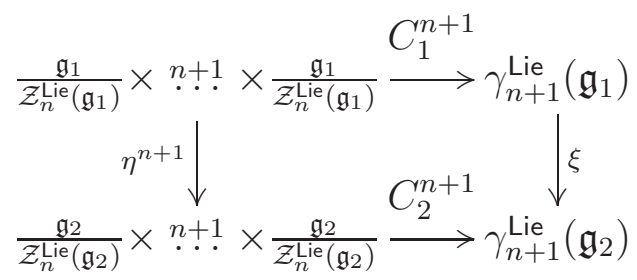

where $C_{1}^{n+1}\left(\bar{x}_{1}, \ldots, \bar{x}_{n+1}\right)=\left[\left[\left[x_{1}, x_{2}\right]_{\text {lie }}, x_{3}\right]_{\text {lie }}, \ldots, x_{n+1}\right]_{\text {lie }}$ with $x_{1}, \ldots, x_{n+1} \in \mathfrak{g}_{1}$ and $\bar{x}:=x+\mathcal{Z}_{n}^{\text {Lie }}\left(\mathfrak{g}_{1}\right)$, and $C_{2}^{n+1}\left(\tilde{y}_{1}, \ldots, \tilde{y}_{n+1}\right)=\left[\left[\left[y_{1}, y_{2}\right]_{l i e}, y_{3}\right]_{\text {lie }}, \ldots, y_{n+1}\right]_{\text {lie }}$ with $y_{1}, \ldots, y_{n+1} \in \mathfrak{g}_{2}$ and $\tilde{y}:=y+\mathcal{Z}_{n}^{\text {Lie }}\left(\mathfrak{g}_{2}\right)$ (see Definitions 3.1 and 3.2).

This paper is organized as follows: In section 2, we present some generalities and preliminaries. In section 3 , we define the notion of $n$-Lie isoclinism between Lie-central extensions of Leibniz algebras and we prove the corresponding relative results and characterizations of several classical results on isoclinism of Lie algebras in this framework such as any Leibniz algebra is $n$-Lie-isoclinic to some Leibniz algebra $\mathfrak{h}$ satisfying $Z_{\text {Lie }}(\mathfrak{h}) \cap \gamma_{n}^{\text {Lie }}(\mathfrak{h}) \subseteq \gamma_{n+1}^{\text {Lie }}(\mathfrak{h})$ or a homomorphism of Lie-central extensions $(\alpha, \beta, \gamma):\left(g_{1}\right) \rightarrow\left(g_{2}\right)$ is $n$-Lie-isoclinic if and only if $\gamma$ is an isomorphism and $\operatorname{Ker}(\beta) \cap \gamma_{n+1}^{\text {Lie }}\left(\mathfrak{g}_{1}\right)=0$. In section 4, we study properties of $n$-Lie isoclinism of Lie-stem Leibniz algebras proving that every Leibniz algebra is $n$-Lie-iscolinic to some $n$-Lie-stem Leibniz algebra or that two $n$-Lie-isoclinic $n$-Lie-stem Leibniz algebras have isomorphic $n$-Lie centers.

\section{Preliminary results on Leibniz algebras}

Let $\mathbb{K}$ be a fix ground field such that $\frac{1}{2} \in \mathbb{K}$. Throughout the paper, all vector spaces and tensor products are considered over $\mathbb{K}$.

A Leibniz algebra [14, 15] is a vector space $\mathfrak{g}$ equipped with a bilinear map $[-,-]: \mathfrak{g} \otimes \mathfrak{g} \rightarrow \mathfrak{g}$, usually called the Leibniz bracket of $\mathfrak{g}$, satisfying the Leibniz identity:

$$
[x,[y, z]]=[[x, y], z]-[[x, z], y], \quad x, y, z \in \mathfrak{g} .
$$


A subalgebra $\mathfrak{h}$ of a Leibniz algebra $\mathfrak{g}$ is said to be left (resp. right) ideal of $\mathfrak{g}$ if $[h, g] \in \mathfrak{h}$ (resp. $[g, h] \in \mathfrak{h}$ ), for all $h \in \mathfrak{h}, g \in \mathfrak{g}$. If $\mathfrak{h}$ is both left and right ideal, then $\mathfrak{h}$ is called two-sided ideal of $\mathfrak{g}$. In this case $\mathfrak{g} / \mathfrak{h}$ naturally inherits a Leibniz algebra structure.

Given a Leibniz algebra $\mathfrak{g}$, we denote by $\mathfrak{g}^{\text {ann }}$ the subspace of $\mathfrak{g}$ spanned by all elements of the form $[x, x], x \in \mathfrak{g}$. It is clear that the quotient $\mathfrak{g}_{\text {Lie }}=\mathfrak{g} / \mathfrak{g}^{\text {ann }}$ is a Lie algebra. This defines the so-called Liezation functor $(-)_{\text {Lie }}$ : Leib $\rightarrow$ Lie, which assigns to a Leibniz algebra $\mathfrak{g}$ the Lie algebra $\mathfrak{g}_{\text {Lie }}$. Moreover, the canonical epimorphism $\mathfrak{g} \rightarrow \mathfrak{g}_{\text {Lie }}$ is universal among all homomorphisms from $\mathfrak{g}$ to a Lie algebra, implying that the Liezation functor is left adjoint to the inclusion functor Lie $\hookrightarrow$ Leib.

Given a Leibniz algebra $\mathfrak{g}$, we define the bracket

$$
[-,-]_{l i e}: \mathfrak{g} \rightarrow \mathfrak{g} \text {, by }[x, y]_{l i e}=[x, y]+[y, x], \text { for } x, y \in \mathfrak{g} .
$$

Let $\mathfrak{m}, \mathfrak{n}$ be two-sided ideals of a Leibniz algebra $\mathfrak{g}$. The following notions come from [7], which were derived from [8].

The Lie-commutator of $\mathfrak{m}$ and $\mathfrak{n}$ is the two-sided ideal of $\mathfrak{g}$

$$
[\mathfrak{m}, \mathfrak{n}]_{\text {Lie }}=\left\langle\left\{[m, n]_{\text {liee }}, m \in \mathfrak{m}, n \in \mathfrak{n}\right\}\right\rangle .
$$

The Lie-center of the Leibniz algebra $\mathfrak{g}$ is the two-sided ideal

$$
Z_{\text {Lie }}(\mathfrak{g})=\left\{z \in \mathfrak{g} \mid[q, z]_{\text {lie }}=0 \text { for all } q \in \mathfrak{g}\right\} .
$$

The Lie-centralizer of $\mathfrak{m}$ and $\mathfrak{n}$ over $\mathfrak{g}$ is

$$
C_{\mathfrak{g}}^{\text {Lie }}(\mathfrak{m}, \mathfrak{n})=\left\{g \in \mathfrak{g} \mid[g, m]_{\text {lie }} \in \mathfrak{n}, \text { for all } m \in \mathfrak{m}\right\} .
$$

Definition 2.1 [7] Let $\mathfrak{n}$ be a two-sided ideal of a Leibniz algebra $\mathfrak{g}$. The lower Lie-central series of $\mathfrak{g}$ relative to $\mathfrak{n}$ is the sequence

$$
\cdots \unlhd \gamma_{i}^{\text {Lie }}(\mathfrak{g}, \mathfrak{n}) \unlhd \cdots \unlhd \gamma_{2}^{\text {Lie }}(\mathfrak{g}, \mathfrak{n}) \unlhd \gamma_{1}^{\text {Lie }}(\mathfrak{g}, \mathfrak{n})
$$

of two-sided ideals of $\mathfrak{g}$ defined inductively by

$$
\gamma_{1}^{\text {Lie }}(\mathfrak{g}, \mathfrak{n})=\mathfrak{n} \quad \text { and } \quad \gamma_{i}^{\text {Lie }}(\mathfrak{g}, \mathfrak{n})=\left[\gamma_{i-1}^{\text {Lie }}(\mathfrak{g}, \mathfrak{n}), \mathfrak{g}\right]_{\text {Lie }}, \quad i \geq 2 .
$$

We use the notation $\gamma_{i}^{\text {Lie }}(\mathfrak{g})$ instead of $\gamma_{i}^{\text {Lie }}(\mathfrak{g}, \mathfrak{g}), 1 \leq i \leq n$.

If $\varphi: \mathfrak{g} \rightarrow \mathfrak{q}$ is a homomorphism of Leibniz such that $\varphi(\mathfrak{m}) \subseteq \mathfrak{n}$, where $\mathfrak{m}$ is a two-sided ideal of $\mathfrak{g}$ and $\mathfrak{n}$ a two-sided ideal of $\mathfrak{q}$, then $\varphi\left(\gamma_{i}^{\text {Lie }}(\mathfrak{g}, \mathfrak{m})\right) \subseteq$ $\gamma_{i}^{\text {Lie }}(\mathfrak{q}, \mathfrak{n}), i \geq 1$.

Definition 2.2 The Leibniz algebra $\mathfrak{g}$ is said to be Lie-nilpotent relative to $\mathfrak{n}$ of class $c$ if $\gamma_{c+1}^{\text {Lie }}(\mathfrak{g}, \mathfrak{n})=0$ and $\gamma_{c}^{\text {Lie }}(\mathfrak{g}, \mathfrak{n}) \neq 0$. 
Definition 2.3 [7] The upper Lie-central series of a Leibniz algebra $\mathfrak{g}$ is the sequence of two-sided ideals, called $i$-Lie centers, $i=0,1,2, \ldots$,

$$
\mathcal{Z}_{0}^{\text {Lie }}(\mathfrak{g}) \unlhd \mathcal{Z}_{1}^{\text {Lie }}(\mathfrak{g}) \unlhd \cdots \unlhd \mathcal{Z}_{i}^{\text {Lie }}(\mathfrak{g}) \unlhd \cdots
$$

defined inductively by

$$
\mathcal{Z}_{0}^{\mathrm{Lie}}(\mathfrak{g})=0 \quad \text { and } \quad \mathcal{Z}_{i}^{\mathrm{Lie}}(\mathfrak{g})=C_{\mathfrak{g}}^{\mathrm{Lie}}\left(\mathfrak{g}, \mathcal{Z}_{i-1}^{\mathrm{Lie}}(\mathfrak{g})\right), \quad i \geq 1
$$

Definition 2.4 [4, Definition 3.2, Proposition 3.4] An exact sequence of Leibniz algebras $0 \rightarrow \mathfrak{n} \rightarrow \mathfrak{g} \stackrel{\pi}{\rightarrow} \mathfrak{q} \rightarrow 0$ is said to be $n$-Lie-central extension if $\gamma_{n+1}^{\text {Lie }}(\mathfrak{g}, \mathfrak{n})=$ 0 , equivalently $\mathfrak{n} \subseteq \mathcal{Z}_{n}^{\text {Lie }}(\mathfrak{g})$.

\section{$3 n$-Lie-isoclinic Leibniz algebras}

Let $\mathfrak{g}_{1}$ and $\mathfrak{g}_{2}$ be two Leibniz algebras, then we can construct the following $n$-Liecentral extensions

$$
\left(g_{i}\right): 0 \rightarrow \mathcal{Z}_{n}^{\text {Lie }}\left(\mathfrak{g}_{i}\right) \rightarrow \mathfrak{g}_{i} \rightarrow \mathfrak{g}_{i} / \mathcal{Z}_{n}^{\text {Lie }}\left(\mathfrak{g}_{i}\right) \rightarrow 0, i=1,2 .
$$

Definition 3.1 The Lie-central extensions $\left(g_{1}\right)$ and $\left(g_{2}\right)$ are said to be $n$-Lieisoclinic whenever there exist two isomorphisms $\eta: \frac{\mathfrak{g}_{1}}{\mathcal{Z}_{n}^{\text {Lie }\left(\mathfrak{g}_{1}\right)}} \rightarrow \frac{\mathfrak{g}_{2}}{\mathcal{Z}_{n}^{\text {Lie }}\left(\mathfrak{g}_{2}\right)}$ and $\xi$ : $\gamma_{n+1}^{\text {Lie }}\left(\mathfrak{g}_{1}\right) \rightarrow \gamma_{n+1}^{\text {Lie }}\left(\mathfrak{g}_{2}\right)$ such that the following diagram is commutative:

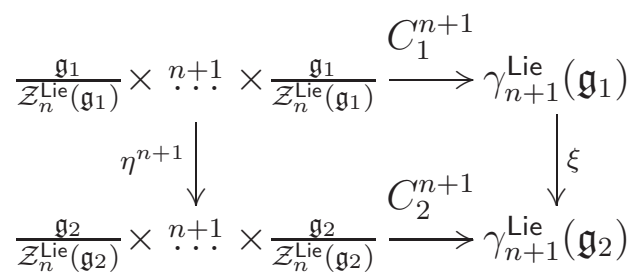

where $C_{1}^{n+1}\left(\bar{x}_{1}, \ldots, \bar{x}_{n+1}\right)=\left[\left[\left[x_{1}, x_{2}\right]_{\text {lie }}, x_{3}\right]_{\text {lie }}, \ldots, x_{n+1}\right]_{\text {lie }}$ with $x_{1}, \ldots, x_{n+1} \in \mathfrak{g}_{1}$ and $\bar{x}:=x+\mathcal{Z}_{n}^{\text {Lie }}\left(\mathfrak{g}_{1}\right)$, and $C_{2}^{n+1}\left(\tilde{y}_{1}, \ldots, \tilde{y}_{n+1}\right)=\left[\left[\left[y_{1}, y_{2}\right]_{\text {lie }}, y_{3}\right]_{\text {lie }}, \ldots, y_{n+1}\right]_{\text {lie }}$ with $y_{1}, \ldots, y_{n+1} \in \mathfrak{g}_{2}$ and $\tilde{y}:=y+\mathcal{Z}_{n}^{\text {Lie }}\left(\mathfrak{g}_{2}\right)$. So the map $\xi$ is given by $\xi\left(\left[\left[\left[x_{1}, x_{2}\right]_{\text {lie }}, x_{3}\right]_{\text {lie }}\right.\right.$, $\left.\left.\ldots, x_{n+1}\right]_{\text {lie }}\right)=\left[\left[\left[y_{1}, y_{2}\right]_{\text {lie }}, y_{3}\right]_{\text {lie }}, \ldots, y_{n+1}\right]_{\text {lie }}$, with $x_{i} \in \mathfrak{g}_{1}$ and $y_{i} \in \eta\left(\bar{x}_{i}\right)$, for $i=1, \ldots, n+1$.

The pair $(\eta, \xi)$ is called a $n$-Lie-isoclinism from $\left(g_{1}\right)$ to $\left(g_{2}\right)$, and will be denoted by $(\eta, \xi):\left(g_{1}\right) \rightarrow\left(g_{2}\right)$.

Definition 3.2 Let $\mathfrak{g}_{1}$ and $\mathfrak{g}_{2}$ be Leibniz algebras. We say that $\mathfrak{g}_{1}$ and $\mathfrak{g}_{2}$ are $n$-Lie-isoclinic if $\left(g_{1}\right)$ and $\left(g_{2}\right)$ are $n$-Lie-isoclinic $n$-Lie-central extensions. We denote it by $(\eta, \xi): \mathfrak{g}_{1} \underset{n}{\sim} \mathfrak{g}_{2}$.

Remark 3.3 Clearly, 0-Lie-isoclinism coincides with isomorphism and1-Lie-isoclinism coincides with the notion of Lie-isoclinism given in [3]. 
Proposition 3.4 n-Lie-isoclinism is an equivalence relation.

Proof. The proof is straightforward

Lemma 3.5 Let $\mathfrak{g}$ be a Leibniz algebra, $\mathfrak{I}$ an ideal of $\mathfrak{g}$, and $\mathfrak{h}$ a subalgebra of $\mathfrak{g}$. Then

a) $\mathfrak{g}$ is $n$-Lie-isoclinic to $\mathfrak{g} \oplus \mathfrak{n}$, for all Lie-nilpotent Leibniz algebra $\mathfrak{n}$ of class $n$.

b) $\mathfrak{h}$ is $n$-Lie-isoclinic to $\mathfrak{h}+\mathcal{Z}_{n}^{\text {Lie }}(\mathfrak{g})$ for $n \geq 1$ provided that $\left[\mathcal{Z}_{n-1}^{\text {Lie }}(\mathfrak{h}), \mathfrak{h}\right]_{\text {Lie }} \subseteq$ $\gamma_{n+1}^{\text {Lie }}(\mathfrak{h})$. Consequently, under the above requirements for $n$ and $\mathfrak{h}$, if $\mathfrak{g}=$ $\mathfrak{h}+\mathcal{Z}_{n}^{\text {Lie }}(\mathfrak{g})$, then $\mathfrak{g}$ is $n$-Lie-isoclinic to $\mathfrak{h}$.

c) If $\mathfrak{g}$ is $n$-Lie-isoclinic to $\mathfrak{h}$ such that $\left[\mathcal{Z}_{n-1}^{\text {Lie }}(\mathfrak{h}), \mathfrak{h}\right]_{\text {Lie }} \subseteq \gamma_{n+1}^{\text {Lie }}(\mathfrak{h})$ and $\mathfrak{g} / \mathcal{Z}_{n}^{\text {Lie }}(\mathfrak{g})$ is finite dimensional, then $\mathfrak{g}=\mathfrak{h}+\mathcal{Z}_{n}^{\text {Lie }}(\mathfrak{g})$.

d) $\frac{\mathfrak{g}}{\mathfrak{J}}$ is $n$-Lie-isoclinic to $\frac{\mathfrak{g}}{\mathfrak{J} \cap \gamma_{n+1}^{\text {Lie }}(\mathfrak{g})}$. Consequently, if $\mathfrak{I} \cap \gamma_{n+1}^{\text {Lie }}(\mathfrak{g})=0$, then $\mathfrak{g}$ is $n$-Lie-isoclinic to $\frac{\mathfrak{g}}{\mathfrak{y}}$.

e) If $\gamma_{n+1}^{\text {Lie }}(\mathfrak{g})$ is a finite dimensional subalgebra of $\mathfrak{g}$ and $\mathfrak{g}$ is $n$-Lie-isoclinic to $\frac{\mathfrak{g}}{\mathfrak{I}}$, then $\mathfrak{I} \cap \gamma_{n+1}^{\operatorname{Lie}}(\mathfrak{g})=0$.

Proof. To prove $a$ ), let $\mathfrak{n}$ be a Lie-nilpotent Leibniz algebra of class $n$. Then $\gamma_{n+1}^{\text {Lie }}(\mathfrak{n})=0$ and $\mathcal{Z}_{n}^{\text {Lie }}(\mathfrak{n})=\mathfrak{n}$. So $\mathcal{Z}_{n}^{\text {Lie }}(\mathfrak{g} \oplus \mathfrak{n})=\mathcal{Z}_{n}^{\text {Lie }}(\mathfrak{g}) \oplus \mathcal{Z}_{n}^{\text {Lie }}(\mathfrak{n})=\mathcal{Z}_{n}^{\text {Lie }}(\mathfrak{g}) \oplus \mathfrak{n}$, and $\gamma_{n+1}^{\text {Lie }}(\mathfrak{g} \oplus \mathfrak{n})=\gamma_{n+1}^{\text {Lie }}(\mathfrak{g}) \oplus \gamma_{n+1}^{\text {Lie }}(\mathfrak{n})=\gamma_{n+1}^{\text {Lie }}(\mathfrak{g})$. Consider the maps $\eta: \frac{\mathfrak{g}}{\mathcal{Z}_{n}^{\text {Lie }}(\mathfrak{g})} \rightarrow \frac{\mathfrak{g} \oplus \mathfrak{n}}{\mathcal{Z}_{n}^{\operatorname{Lie}(\mathfrak{g}) \oplus \mathfrak{n}}}$ and $\xi=i d_{\gamma_{n+1}^{\text {Lie }}(\mathfrak{g})}$, where $\eta\left(g+\mathcal{Z}_{n}^{\text {Lie }}(\mathfrak{g})\right)=g+\left(\mathcal{Z}_{n}^{\text {Lie }}(\mathfrak{g}) \oplus \mathfrak{n}\right)$. It is easy to check that $\eta$ and $\xi$ are isomorphisms and the corresponding diagram (2) commutes.

To prove $b$ ), set $\mathfrak{h}_{1}=\mathfrak{h}+\mathcal{Z}_{n}^{\text {Lie }}(\mathfrak{g})$ and consider the mapping $\eta: \mathfrak{h} / \mathcal{Z}_{n}^{\text {Lie }}(\mathfrak{h}) \rightarrow$ $\mathfrak{h}_{1} / \mathcal{Z}_{n}^{\text {Lie }}\left(\mathfrak{h}_{1}\right)$ defined by $\eta\left(h+\mathcal{Z}_{n}^{\text {Lie }}(\mathfrak{h})\right)=h+\mathcal{Z}_{n}^{\text {Lie }}\left(\mathfrak{h}_{1}\right) . \eta$ is clearly a surjective homomorphism. To show that $\eta$ is one-to-one, it is enough to show that $\mathfrak{h} \cap$ $\mathcal{Z}_{n}^{\text {Lie }}\left(\mathfrak{h}_{1}\right) \subseteq \mathcal{Z}_{n}^{\text {Lie }}(\mathfrak{h})$. We proceed by induction. Clearly, for $h \in \mathfrak{h} \cap \mathcal{Z}_{1}^{\text {Lie }}\left(\mathfrak{h}_{1}\right)$, $[h, x]_{\text {lie }}=0$ for all $x \in \mathfrak{h}$ since $\mathfrak{h} \subseteq \mathfrak{h}_{1}$, so $\mathfrak{h} \cap \mathcal{Z}_{1}^{\text {Lie }}\left(\mathfrak{h}_{1}\right) \subseteq \mathcal{Z}_{1}^{\text {Lie }}(\mathfrak{h})$. Now let $h \in$ $\mathfrak{h} \cap \mathcal{Z}_{n}^{\text {Lie }}\left(\mathfrak{h}_{1}\right)$, then for all $z \in \mathfrak{h} \subseteq \mathfrak{h}_{1},[h, z]_{l i e} \in \mathfrak{h} \cap \mathcal{Z}_{n-1}^{\text {Lie }}\left(\mathfrak{h}_{1}\right) \subseteq \mathcal{Z}_{n-1}^{\text {Lie }}(\mathfrak{h})$, implying that $h \in \mathcal{Z}_{n}^{\text {Lie }}(\mathfrak{h})$. So $\mathfrak{h} \cap \mathcal{Z}_{n}^{\text {Lie }}\left(\mathfrak{h}_{1}\right) \subseteq \mathcal{Z}_{n}^{\text {Lie }}(\mathfrak{h})$. Therefore $\eta$ is an isomorphism.

Then we claim that $(\eta, i d):\left(h_{1}\right) \underset{n}{\sim}(h)$ is a $n$-Lie-isoclinism, with the identity mapping $i d: \gamma_{n}^{\text {Lie }}(\mathfrak{h}) \rightarrow \gamma_{n}^{\text {Lie }}\left(\mathfrak{h}_{1}\right)$. Indeed, a standard induction shows that 
$\gamma_{k}^{\text {Lie }}\left(\mathfrak{h}_{1}\right) \subseteq \gamma_{k}^{\text {Lie }}(\mathfrak{h})+\mathcal{Z}_{n-1}^{\text {Lie }}(\mathfrak{g})$ for all $2 \leq k \leq n$. We now have

$$
\begin{aligned}
\gamma_{n+1}^{\text {Lie }}\left(\mathfrak{h}_{1}\right) & =\left[\gamma_{n}^{\text {Lie }}\left(\mathfrak{h}_{1}\right), \mathfrak{h}_{1}\right]_{\text {Lie }} \\
& =\left[\gamma_{n}^{\text {Lie }}\left(\mathfrak{h}_{1}\right), \mathfrak{h}+\mathcal{Z}_{n}^{\text {Lie }}(\mathfrak{g})\right]_{\text {Lie }} \\
& =\left[\gamma_{n}^{\text {Lie }}\left(\mathfrak{h}_{1}\right), \mathfrak{h}\right]_{\text {Lie }} \operatorname{since}\left[\gamma_{n}^{\text {Lie }}\left(\mathfrak{h}_{1}\right), \mathcal{Z}_{n}^{\text {Lie }}(\mathfrak{g})\right]_{\text {Lie }}=0 \\
& \subseteq\left[\gamma_{n}^{\text {Lie }}(\mathfrak{h})+\mathcal{Z}_{n-1}^{\text {Lie }}(\mathfrak{g}), \mathfrak{h}\right]_{\text {Lie }} \\
& \subseteq\left[\gamma_{n}^{\text {Lie }}(\mathfrak{h}), \mathfrak{h}\right]_{\text {Lie }}+\left[\mathcal{Z}_{n-1}^{\text {Lie }}(\mathfrak{g}), \mathfrak{h}\right]_{\text {Lie }} \\
& \subseteq \gamma_{n+1}^{\text {Lie }}(\mathfrak{h})+\left[\mathcal{Z}_{n-1}^{\text {Lie }}(\mathfrak{h}), \mathfrak{h}\right]_{\text {Lie }} \\
& \subseteq \gamma_{n+1}^{\text {Lie }}(\mathfrak{h}) .
\end{aligned}
$$

The converse inclusion is obvious and the commutativity of diagram (2) is trivial.

To prove $c$ ), assume that $\mathfrak{g} \underset{n}{\sim} \mathfrak{h}$ and $\operatorname{dim}\left(\frac{\mathfrak{g}}{\mathcal{Z}_{n}^{\text {Lie }}(\mathfrak{g})}\right)$ is finite. Again, set $\mathfrak{h}_{1}=$ $\mathfrak{h}+\mathcal{Z}_{n}^{\text {Lie }}(\mathfrak{g})$. Since by b), $\mathfrak{h} \underset{n}{\sim} \mathfrak{h}_{1}$, we have by Proposition 3.4 that $\mathfrak{g} \underset{n}{\sim} \mathfrak{h}_{1}$ which implies that $\frac{\mathfrak{g}}{\mathcal{Z}_{n}^{\text {Lie }(\mathfrak{g})}} \stackrel{\eta}{\cong} \frac{\mathfrak{h}_{1}}{\mathcal{Z}_{n}^{\text {Lie }\left(\mathfrak{h}_{1}\right)}}$. As $\mathcal{Z}_{n}^{\text {Lie }}(\mathfrak{g}) \subseteq \mathcal{Z}_{n}^{\text {Lie }}\left(\mathfrak{h}_{1}\right)$, it follows that

$$
\operatorname{dim}\left(\frac{\mathfrak{g}}{\mathcal{Z}_{n}^{\mathrm{Lie}}(\mathfrak{g})}\right)=\operatorname{dim}\left(\frac{\mathfrak{h}_{1}}{\mathcal{Z}_{n}^{\mathrm{Lie}}\left(\mathfrak{h}_{1}\right)}\right) \leq \operatorname{dim}\left(\frac{\mathfrak{g}}{\mathcal{Z}_{n}^{\mathrm{Lie}}\left(\mathfrak{h}_{1}\right)}\right) \leq \operatorname{dim}\left(\frac{\mathfrak{g}}{\mathcal{Z}_{n}^{\mathrm{Lie}}(\mathfrak{g})}\right)
$$

Since $\operatorname{dim}\left(\frac{\mathfrak{g}}{\left.\mathcal{Z}_{n}^{\text {Lie(g) }}\right)}\right)$ is finite, it follows that $\mathfrak{g} \cong \mathfrak{h}_{1}=\mathfrak{h}+\mathcal{Z}_{n}^{\text {Lie }}(\mathfrak{g})$.

To prove d), consider the map $\eta: \mathfrak{g}_{1} / \mathcal{Z}_{n}^{\text {Lie }}\left(\mathfrak{g}_{1}\right) \rightarrow \mathfrak{g}_{2} / \mathcal{Z}_{n}^{\text {Lie }}\left(\mathfrak{g}_{2}\right)$ where $\mathfrak{g}_{1}=$ $\frac{\mathfrak{g}}{\mathfrak{I}}$ and $\mathfrak{g}_{2}=\frac{\mathfrak{g}}{\mathfrak{I} \cap \gamma_{n+1}^{\text {Lie }}(\mathfrak{g})}$, defined by $\eta\left(\bar{g}+\mathcal{Z}_{n}^{\text {Lie }}\left(\mathfrak{g}_{1}\right)\right)=\tilde{g}+\mathcal{Z}_{n}^{\text {Lie }}\left(\mathfrak{g}_{2}\right)$ with $\bar{g}=$ $g+\mathfrak{I}$ and $\tilde{g}=g+\mathfrak{I} \cap \gamma_{n+1}^{\text {Lie }}(\mathfrak{g})$. Then $\eta$ is clearly an isomorphism. Also the $\operatorname{map} \xi: \gamma_{n+1}^{\text {Lie }}\left(\mathfrak{g}_{1}\right) \rightarrow \gamma_{n+1}^{\text {Lie }}\left(\mathfrak{g}_{2}\right)$ defined by $\xi\left(\left[\left[\left[\bar{g}_{1}, \bar{g}_{2}\right]_{l i e}, \bar{g}_{3}\right]_{l i e}, \ldots, \bar{g}_{n+1}\right]_{l i e}\right)=$ $\left[\left[\left[\tilde{g}_{1}, \tilde{g}_{2}\right]_{l i e}, \tilde{g}_{3}\right]_{l i e}, \ldots, \tilde{g}_{n+1}\right]_{l i e}, g_{1}, \ldots, g_{n+1} \in \mathfrak{g}$, is a well-defined isomorphism and the corresponding diagram (2) commutes. Therefore $(\eta, \xi)$ is a $n$-Lie-isoclinism.

To prove $e$ ), assume that $\operatorname{dim}\left(\gamma_{n+1}^{\operatorname{Lie}}(\mathfrak{g})\right)$ is finite and $\mathfrak{g} \underset{n}{\sim} \frac{\mathfrak{g}}{\mathfrak{J}}$. Then $\gamma_{n+1}^{\text {Lie }}(\mathfrak{g}) \stackrel{\xi}{\cong}$ $\gamma_{n+1}^{\text {Lie }}\left(\frac{\mathfrak{g}}{\mathfrak{I}}\right)$. Also, by $d$ ) we have $\frac{\mathfrak{g}}{\mathfrak{I}} \underset{n}{\sim} \frac{\mathfrak{g}}{\mathfrak{I} \cap \gamma_{n+1}^{\text {Lie }}(\mathfrak{g})}$ which yields $\gamma_{n+1}^{\text {Lie }}\left(\frac{\mathfrak{g}}{\mathfrak{I}}\right) \stackrel{\xi^{\prime}}{\cong} \gamma_{n+1}^{\text {Lie }}\left(\frac{\mathfrak{g}}{\mathfrak{I} \cap \gamma_{n+1}^{\text {Lie }(\mathfrak{g})}}\right) \cong$ $\frac{\gamma_{n+1}^{\text {Lie }}(\mathfrak{g})}{\mathfrak{I} \cap \gamma_{n+1}^{\text {Lie }}(\mathfrak{g})}$. Therefore $\gamma_{n+1}^{\text {Lie }}(\mathfrak{g}) \cong \frac{\gamma_{n+1}^{\text {Lie }}(\mathfrak{g})}{\mathfrak{I} \cap \gamma_{n+1}^{\operatorname{Lie}}(\mathfrak{g})}$. Hence $\mathfrak{I} \cap \gamma_{n+1}^{\text {Lie }}(\mathfrak{g})=0$ as $\operatorname{dim}\left(\gamma_{n+1}^{\text {Lie }}(\mathfrak{g})\right)$ is finite.

Example 3.6 An example of the requirements in Lemma 3.5 b) is given by the four-dimensional non-Lie-nilpotent non-Lie Leibniz algebra $\mathfrak{h}=\operatorname{span}\left\{a_{1}, a_{2}, a_{3}, a_{4}\right\}$ with nonzero brackets $\left[a_{1}, a_{1}\right]=a_{4}, \quad\left[a_{1}, a_{2}\right]=-a_{2}, \quad\left[a_{2}, a_{1}\right]=a_{2}, \quad\left[a_{3}, a_{1}\right]=$ $-a_{3}, \quad\left[a_{3}, a_{2}\right]=a_{4}$ (algebra $\mathcal{L}_{5}$ in [5, Proposition 3.9]).

Then clearly, $\mathcal{Z}_{n}^{\text {Lie }}(\mathfrak{h})=\operatorname{span}\left\{a_{2}, a_{4}\right\}$, for all $n \geq 1$. Also, $\gamma_{n}^{\text {Lie }}(\mathfrak{h})=\operatorname{span}\left\{a_{3}, a_{4}\right\}$ for all $n \geq 2$. One easily verifies that $\left[\mathcal{Z}_{0}^{\text {Lie }}(\mathfrak{h}), \mathfrak{h}\right]_{\text {Lie }}=0 \subseteq \gamma_{2}^{\text {Lie }}(\mathfrak{h}), \quad\left[\mathcal{Z}_{1}^{\text {Lie }}(\mathfrak{h}), \mathfrak{h}\right]_{\text {Lie }}=$ $0 \subseteq \gamma_{3}^{\text {Lie }}(\mathfrak{h}) \quad$ and $\quad\left[\mathcal{Z}_{n-1}^{\text {Lie }}(\mathfrak{h}), \mathfrak{h}\right]_{\text {Lie }}=\operatorname{span}\left\{a_{4}\right\} \subseteq \gamma_{n+1}^{\text {Lie }}(\mathfrak{h})$ for all $n>2$ 
Remark 3.7 Lemma 3.5 b) in case $n=1$ provides the isoclinism given in Proposition $3.20 \mathrm{c}$ ) in [3].

Corollary 3.8 Let $\mathfrak{g}$ be a Leibniz algebra. Then $\mathfrak{g}$ is $n$-Lie-isoclinic to some Leibniz algebra $\mathfrak{h}$ satisfying $Z_{\text {Lie }}(\mathfrak{h}) \cap \gamma_{n}^{\text {Lie }}(\mathfrak{h}) \subseteq \gamma_{n+1}^{\text {Lie }}(\mathfrak{h})$.

Proof. Let $\gamma=\left(\gamma_{n}^{\text {Lie }}(\mathfrak{g}) \backslash \gamma_{n+1}^{\text {Lie }}(\mathfrak{g})\right)$. Then $\mathfrak{t}_{n}:=\gamma \cap Z_{\text {Lie }}(\mathfrak{g})$ is a two-sided ideal of $\mathfrak{g}$ satisfying $\mathfrak{t}_{n} \cap \gamma_{n+1}^{\text {Lie }}(\mathfrak{g})=0$. Therefore $\mathfrak{g} / \mathfrak{t}_{n}$ is $n$-Lie-isoclinic to $\mathfrak{g}$ by Lemma 3.5 $d$ ). Take $\mathfrak{h}:=\mathfrak{g} / \mathfrak{t}_{n}$. It remains to show that $Z_{\text {Lie }}(\mathfrak{h}) \cap \gamma_{n}^{\text {Lie }}(\mathfrak{h}) \subseteq \gamma_{n+1}^{\text {Lie }}(\mathfrak{h})$. Indeed, let $x+\mathfrak{t}_{n} \in Z_{\text {Lie }}(\mathfrak{h}) \cap \gamma_{n}^{\text {Lie }}(\mathfrak{h})=\frac{Z_{\text {Lie }}(\mathfrak{g})}{\mathfrak{t}_{n}} \cap \frac{\gamma_{n}^{\text {Lie }}(\mathfrak{g})}{\mathfrak{t}_{n}}$. Without loss of generality, assume that $x \in \gamma_{n}^{\text {Lie }}(\mathfrak{g})$. Then $y:=[x, g]_{l i e} \in \mathfrak{t}_{n}$ for all $g \in \mathfrak{g}$, and $y \in \gamma_{n+1}^{\text {Lie }}(\mathfrak{g})$. So $y=0$ since $\mathfrak{t}_{n} \cap \gamma_{n+1}^{\text {Lie }}(\mathfrak{g})=0$. This implies that $x \in Z_{\text {Lie }}(\mathfrak{g})$, and so $x \in \gamma_{n}^{\text {Lie }}(\mathfrak{g}) \cap Z_{\text {Lie }}(\mathfrak{g})=$ $\gamma_{n+1}^{\text {Lie }}(\mathfrak{g}) \cap Z_{\text {Lie }}(\mathfrak{g})+\mathfrak{t}_{n}$, and thus $x+\mathfrak{t}_{n} \in \gamma_{n+1}^{\text {Lie }}(\mathfrak{h})$ since $\gamma_{n+1}^{\text {Lie }}(\mathfrak{h})=\frac{\gamma_{n+1}^{\text {Lie }}(\mathfrak{g})}{\mathfrak{t}_{n}}$.

Proposition 3.9 Let $(\eta, \xi):\left(g_{1}\right) \underset{n}{\sim}\left(g_{2}\right)$ be a $n$-Lie-isoclinism and consider the following set

$$
\mathfrak{g}_{2}^{\eta}=\left\{\left(g, x+\mathcal{Z}_{n}^{\mathrm{Lie}}\left(\mathfrak{g}_{1}\right)\right) \in \mathfrak{g}_{2} \times \frac{\mathfrak{g}_{1}}{\mathcal{Z}_{n}^{\mathrm{Lie}}\left(\mathfrak{g}_{1}\right)} \mid g+\mathcal{Z}_{n}^{\mathrm{Lie}}\left(\mathfrak{g}_{2}\right)=\eta\left(x+\mathcal{Z}_{n}^{\mathrm{Lie}}\left(\mathfrak{g}_{1}\right)\right)\right\} .
$$

There is a $n$-Lie-central extension $\left(\eta^{*}\left(g_{2}\right)\right): 0 \rightarrow \mathcal{Z}_{n}^{\mathrm{Lie}}\left(\mathfrak{g}_{2}\right) \rightarrow \mathfrak{g}_{2}^{\eta} \stackrel{\bar{\pi}_{2}}{\rightarrow} \frac{\mathfrak{g}_{1}}{\mathcal{Z}_{n}^{\mathrm{Lie}}\left(\mathfrak{g}_{1}\right)} \rightarrow 0$ isomorphic to $\left(g_{2}\right)$, and $n$-Lie-isoclinic to $\left(g_{1}\right)$.

Proof. Choose $\eta^{\prime}: i d_{\mathfrak{g}_{1} / \mathcal{Z}_{n}^{\text {Lie }\left(\mathfrak{g}_{1}\right)}}$ the identity map, and define $\xi^{\prime}: \gamma_{n+1}^{\text {Lie }}\left(\mathfrak{g}_{1}\right) \rightarrow$ $\gamma_{n+1}^{\text {Lie }}\left(\mathfrak{g}_{2}^{\eta}\right)$ by $\xi^{\prime}\left(C_{1}^{n+1}\left(\bar{x}_{1}, \ldots, \bar{x}_{n+1}\right)\right)=C_{2}^{\eta}\left(\bar{x}_{1}, \ldots, \bar{x}_{n+1}\right)$ where

$$
C_{2}^{\eta}\left(\bar{x}_{1}, \ldots, \bar{x}_{n+1}\right)=\left(\xi\left(C_{1}^{n+1}\left(\bar{x}_{1}, \ldots, \bar{x}_{n+1}\right)\right),\left[\left[\left[\bar{x}_{1}, \bar{x}_{2}\right]_{l i e}, \ldots, \bar{x}_{n}\right]_{l i e}, \bar{x}_{n+1}\right]_{l i e}\right) .
$$

Then $\xi^{\prime}$ is clearly an isomorphism since $\xi: \gamma_{n+1}^{\text {Lie }}\left(\mathfrak{g}_{1}\right) \rightarrow \gamma_{n+1}^{\text {Lie }}\left(\mathfrak{g}_{2}\right)$ is an isomorphism, and the diagram

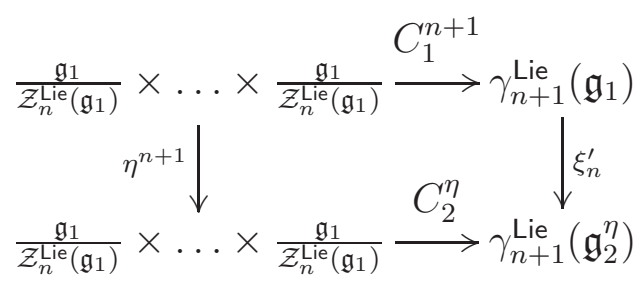

is commutative by construction.

Proposition 3.10 For a $n$-Lie-isoclinism $(\eta, \xi):\left(g_{1}\right) \underset{n}{\sim}\left(g_{2}\right)$, the following statements hold:

a) $\xi(g)+\mathcal{Z}_{n}^{\text {Lie }}\left(\mathfrak{g}_{2}\right)=\eta\left(g+\mathcal{Z}_{n}^{\text {Lie }}\left(\mathfrak{g}_{1}\right)\right)$, for all $g \in \gamma_{n+1}^{\text {Lie }}\left(\mathfrak{g}_{1}\right)$. 
b) $\xi\left(\mathcal{Z}_{n}^{\text {Lie }}\left(\mathfrak{g}_{1}\right) \cap \gamma_{n+1}^{\text {Lie }}\left(\mathfrak{g}_{1}\right)\right)=\mathcal{Z}_{n}^{\text {Lie }}\left(\mathfrak{g}_{2}\right) \cap \gamma_{n+1}^{\text {Lie }}\left(\mathfrak{g}_{2}\right)$.

c) $\xi\left(C_{1}^{n+1}\left(\bar{x}_{1}, \ldots, \bar{x}_{n}, \bar{g}\right)\right)=C_{2}^{n+1}\left(\widetilde{\xi\left(x_{1}\right)}, \ldots, \widetilde{\xi\left(x_{n}\right)}, \tilde{h}\right)$, for all $g \in \mathfrak{g}_{1}, h \in \mathfrak{g}_{2}$ with $\eta(\bar{g})=\tilde{h}$, and $x_{i} \in \mathfrak{g}_{1}, i=1, \ldots, n$, where $\bar{g}=g+\mathcal{Z}_{n}^{\text {Lie }}\left(\mathfrak{g}_{1}\right), \bar{x}_{i}=$ $x_{i}+\mathcal{Z}_{n}^{\text {Lie }}\left(\mathfrak{g}_{1}\right), \widetilde{\xi\left(x_{i}\right)}=\xi\left(x_{i}\right)+\mathcal{Z}_{n}^{\text {Lie }}\left(\mathfrak{g}_{2}\right), i=1, \ldots, n$, and $\tilde{h}=h+\mathcal{Z}_{n}^{\text {Lie }}\left(\mathfrak{g}_{2}\right)$.

\section{Proof.}

a) Let $g \in \gamma_{n+1}^{\text {Lie }}\left(\mathfrak{g}_{1}\right)$. By Proposition [3.9, $\left(i d_{\mathfrak{g}_{1} / \mathcal{Z}_{n}^{\text {Lie }}\left(\mathfrak{g}_{1}\right)}, \xi^{\prime}\right):\left(g_{1}\right) \sim\left(\eta^{*} g_{2}\right)$ is a $n$-Lie-isoclinism where $\left(\xi(g), g+\mathcal{Z}_{n}^{\text {Lie }}\left(\mathfrak{g}_{1}\right)\right) \in \gamma_{n+1}^{\text {Lie }}\left(\mathfrak{g}_{2}^{\eta}\right)$ for all $g \in \gamma_{n+1}^{n}\left(\mathfrak{g}_{1}\right) \subseteq \mathfrak{g}_{2}^{\eta}$. The result follows by definition of $\mathfrak{g}_{2}^{\eta}$.

b) Let $g \in \mathcal{Z}_{n}^{\text {Lie }}\left(\mathfrak{g}_{1}\right) \cap \gamma_{n+1}^{\text {Lie }}\left(\mathfrak{g}_{1}\right)$. Then $\xi(g) \in \gamma_{n+1}^{\text {Lie }}\left(\mathfrak{g}_{2}\right)$. Assume that $\xi(g) \notin$ $\mathcal{Z}_{n}^{\text {Lie }}\left(\mathfrak{g}_{2}\right)$. Then $\xi(g)+\mathcal{Z}_{n}^{\text {Lie }}\left(\mathfrak{g}_{2}\right) \neq \mathcal{Z}_{n}^{\text {Lie }}\left(\mathfrak{g}_{2}\right)$. So by a), $\eta\left(g+\mathcal{Z}_{n}^{\text {Lie }}\left(\mathfrak{g}_{1}\right)\right) \neq \mathcal{Z}_{n}^{\text {Lie }}\left(\mathfrak{g}_{2}\right)$, i.e. $g+\mathcal{Z}_{n}^{\text {Lie }}\left(\mathfrak{g}_{1}\right) \notin \operatorname{Ker}(\eta)=0$ i.e. $g \notin \mathcal{Z}_{n}^{\text {Lie }}\left(\mathfrak{g}_{1}\right)$. A contradiction. So $\xi(g) \in$ $\mathcal{Z}_{n}^{\text {Lie }}\left(\mathfrak{g}_{2}\right) \cap \gamma_{n+1}^{\text {Lie }}\left(\mathfrak{g}_{2}\right)$.

Conversely, if $h \in \mathcal{Z}_{n}^{\text {Lie }}\left(\mathfrak{g}_{2}\right) \cap \gamma_{n+1}^{\text {Lie }}\left(\mathfrak{g}_{2}\right)$, then $h=\xi(g)$ for some $g \in \gamma_{n+1}^{\text {Lie }}\left(\mathfrak{g}_{1}\right)$ since $\xi$ is onto. It follows by a) that $\eta\left(g+\mathcal{Z}_{n}^{\text {Lie }}\left(\mathfrak{g}_{1}\right)\right)=\xi(g)+\mathcal{Z}_{n}^{\text {Lie }}\left(\mathfrak{g}_{2}\right)=h+$ $\mathcal{Z}_{n}^{\text {Lie }}\left(\mathfrak{g}_{2}\right)=\mathcal{Z}_{n}^{\text {Lie }}\left(\mathfrak{g}_{2}\right)$, which implies that $g \in \mathcal{Z}_{n}^{\text {Lie }}\left(\mathfrak{g}_{1}\right)$ since $\eta$ is one-to-one, and thus $g \in \mathcal{Z}_{n}^{\text {Lie }}\left(\mathfrak{g}_{1}\right) \cap \gamma_{n+1}^{\text {Lie }}\left(\mathfrak{g}_{1}\right)$.

c) Since $(\eta, \xi)$ is a $n$-Lie-isoclinism, then the commutativity of diagram (2) provides:

$$
\begin{aligned}
\xi\left(C_{1}^{n+1}\left(\bar{x}_{1}, \ldots, \bar{x}_{n}, \bar{g}\right)\right) & =C_{2}^{n+1}\left(\eta^{n+1}\left(\bar{x}_{1}, \ldots, \bar{x}_{n}, \bar{g}\right)\right) \\
& =C_{2}^{n+1}\left(\eta\left(\bar{x}_{1}\right), \ldots, \eta\left(\bar{x}_{n}\right), \eta(\bar{g})\right) \\
& =C_{2}^{n+1}\left(\widetilde{\xi\left(x_{1}\right)}, \ldots, \widehat{\xi\left(x_{n}\right)}, \tilde{h}\right) .
\end{aligned}
$$

For a $n$-Lie-isoclinism $(\eta, \xi):\left(g_{1}\right) \underset{n}{\sim}\left(g_{2}\right)$, consider the following sets:

$$
\begin{gathered}
\mathcal{K}=\left\{(g, h) \in \mathfrak{g}_{1} \oplus \mathfrak{g}_{2} / \eta\left(g+\mathcal{Z}_{n}^{\text {Lie }}\left(\mathfrak{g}_{1}\right)\right)=h+\mathcal{Z}_{n}^{\text {Lie }}\left(\mathfrak{g}_{2}\right)\right\} \\
Z_{\mathfrak{g}_{1}}=\left\{(g, 0) / g \in \mathcal{Z}_{n}^{\text {Lie }}\left(\mathfrak{g}_{1}\right)\right\} \quad \text { and } \quad Z_{\mathfrak{g}_{2}}=\left\{(0, h) / h \in \mathcal{Z}_{n}^{\text {Lie }}\left(\mathfrak{g}_{2}\right)\right\}
\end{gathered}
$$

Lemma 3.11 The following assertions are true:

a) $\mathcal{K}$ is a subalgebra of $\mathfrak{g}_{1} \times \mathfrak{g}_{2}$.

b) $\gamma_{n+1}^{\text {Lie }}(\mathcal{K})=\left\{(g, \xi(g)) \mid g \in \gamma_{n+1}^{\text {Lie }}\left(\mathfrak{g}_{1}\right)\right\}$.

c) $Z_{\mathfrak{g}_{i}}, i=1,2$ are two-sided ideals of $\mathcal{K}$ satisfying $Z_{\mathfrak{g}_{i}} \cap \gamma_{n+1}^{\text {Lie }}(\mathcal{K})=0$.

d) $\mathfrak{g}_{i} \cong \mathcal{K} / Z_{\mathfrak{g}_{j}} \underset{n}{\sim} \mathcal{K}, \quad i, j=1,2$ with $i \neq j$;

e) $\mathcal{K} / Z_{\mathfrak{g}_{j}} \sim \mathcal{K} / Z_{\mathfrak{g}_{i}} \oplus \mathcal{K} / \gamma_{n+1}^{\text {Lie }}(\mathcal{K})$, provided that $\left[\mathcal{Z}_{n-1}^{\text {Lie }}\left(\mathfrak{g}_{i}\right), \mathfrak{g}_{i}\right]_{\text {Lie }} \subseteq \gamma_{n+1}^{\text {Lie }}\left(\mathfrak{g}_{i}\right)$ for $i=1,2$. 
Proof. The proof of $a$ ) is straightforward. b) follows from the property a) of Proposition 3.10.

For $c$ ), it is easy to check that $Z_{\mathfrak{g}_{i}}, i=1,2$, are two-sided ideals of $\mathcal{K}$ since $\mathcal{Z}_{n}^{\text {Lie }}\left(\mathfrak{g}_{i}\right)$ are two-sided ideals of $\mathfrak{g}_{i}, i=1,2$. That $Z_{\mathfrak{g}_{i}} \cap \gamma_{n+1}^{\text {Lie }}(\mathcal{K})=0, i=1,2$, is due to $\xi$ being one-to-one.

To prove $d)$, it is easy to check that the maps $\tau_{i}: \mathcal{K} \rightarrow \mathfrak{g}_{i}$ defined by $\tau_{i}\left(g_{1}, g_{2}\right)=$ $g_{i}$ are surjective homomorphisms with $\operatorname{kernel} \operatorname{Ker}\left(\tau_{i}\right)=Z_{\mathfrak{g}_{j}}, i, j=1,2$ with $i \neq j$. The isomorphisms follow by the first isomorphism theorem. The isoclinisms follow from Lemma 3.5 $d$ ) since $Z_{\mathfrak{g}_{i}} \cap \gamma_{n+1}^{\text {Lie }}(\mathcal{K})=0, i=1,2$, by statement $c$ ).

To prove $e$ ), consider the sets $\mathcal{K}_{\mathfrak{g}_{i}}=\left\{\left(k+Z_{\mathfrak{g}_{i}}, k+\gamma_{n+1}^{\text {Lie }}(\mathcal{K})\right) / k \in \mathcal{K}\right\}, i=$ 1,2. Then it is clear that $\mathcal{K}_{\mathfrak{g}_{i}}$ are subalgebras of $\mathcal{K} / Z_{\mathfrak{g}_{i}} \oplus \mathcal{K} / \gamma_{n+1}^{\text {Lie }}(\mathcal{K})$ and the mappings $\alpha_{i}: \mathcal{K} \rightarrow \mathcal{K}_{\mathfrak{g}_{i}}$ defined by $\alpha_{i}(k)=\left(k+Z_{\mathfrak{g}_{i}}, k+\gamma_{n+1}^{\text {Lie }}(\mathcal{K})\right)$ are surjective homomorphisms by definition, and one-to-one due to $Z_{\mathfrak{g}_{i}} \cap \gamma_{n+1}^{\text {Lie }}(\mathcal{K})=0$. So $\mathcal{K}$ is isomorphic to $\mathcal{K}_{\mathfrak{g}_{i}}, i=1,2$.

Now apply Lemma 3.5 b) with $\mathfrak{g}:=\mathcal{K} / Z_{\mathfrak{g}_{i}} \oplus \mathcal{K} / \gamma_{n+1}^{\text {Lie }}(\mathcal{K})$, and $\mathfrak{h}:=\mathcal{K}_{\mathfrak{g}_{i}}$, then statement $d$ ) concludes the proof.

Keep in mind that Lemma 3.5 b) is well applied since the hypotheses imply the requirements of Lemma $3.5 \mathrm{~b}$ ). Indeed, for the case $i=1$, assume that $\left[\mathcal{Z}_{n-1}^{\text {Lie }}\left(\mathfrak{g}_{1}\right), \mathfrak{g}_{1}\right]_{\text {Lie }} \subseteq \gamma_{n+1}^{\text {Lie }}\left(\mathfrak{g}_{1}\right)$, and let $k=(g, h) \in \mathcal{K}$ such that $\left(k+Z_{\mathfrak{g}_{1}}, k+\right.$ $\left.\gamma_{n+1}^{\text {Lie }}(\mathcal{K})\right) \in \mathcal{Z}_{n-1}^{\text {Lie }}\left(\mathcal{K}_{\mathfrak{g}_{1}}\right)$. This implies that for every $g_{1}, \ldots, g_{n-1} \in \mathfrak{g}_{1}$, and appropriate $h_{1}, \ldots, h_{n-1} \in \mathfrak{g}_{2}$ so that $k_{1}, \ldots, k_{n-1} \in \mathcal{K}$ with $k_{i}=\left(g_{i}, h_{i}\right)$, we have

$$
\left[\left[\left[k, k_{1}\right]_{l i e}, k_{2}\right]_{l i e}, \ldots, k_{n-1}\right]_{l i e} \in Z_{\mathfrak{g}_{1}} \cap \gamma_{n+1}^{\text {Lie }}(\mathcal{K})=0,
$$

and thus

$$
\left(\left[\left[\left[g, g_{1}\right]_{l i e}, g_{2}\right]_{l i e}, \ldots, g_{n-1}\right]_{l i e},\left[\left[\left[h, h_{1}\right]_{l i e}, h_{2}\right]_{l i e}, \ldots, h_{n-1}\right]_{l i e}\right)=0 .
$$

So $\left[\left[\left[g, g_{1}\right]_{l i e}, g_{2}\right]_{l i e}, \ldots, g_{n-1}\right]_{l i e}=0$ which implies that $g \in \mathcal{Z}_{n-1}^{\text {Lie }}\left(\mathfrak{g}_{1}\right)$. Therefore, for all $k^{\prime}=\left(g^{\prime}, h^{\prime}\right) \in \mathcal{K},\left[\left(k+Z_{\mathfrak{g}_{1}}, k+\gamma_{n+1}^{\text {Lie }}(\mathcal{K})\right),\left(k^{\prime}+Z_{\mathfrak{g}_{1}}, k^{\prime}+\gamma_{n+1}^{\text {Lie }}(\mathcal{K})\right)\right]_{l i e}=$ $\left(\left(\left[g, g^{\prime}\right]_{l i e},\left[h, h^{\prime}\right]_{l i e}\right)+Z_{\mathfrak{g}_{1}},\left(\left[g, g^{\prime}\right]_{l i e},\left[h, h^{\prime}\right]_{l i e}\right)+\gamma_{n+1}^{\text {Lie }}(\mathcal{K})\right) \in \gamma_{n+1}^{\text {Lie }}\left(\mathcal{K}_{\mathfrak{g}_{1}}\right)$ since $\left[g, g^{\prime}\right]_{l i e} \in$ $\left[\mathcal{Z}_{n-1}^{\text {Lie }}\left(\mathfrak{g}_{1}\right), \mathfrak{g}_{1}\right]_{\text {Lie }} \subseteq \gamma_{n+1}^{\text {Lie }}\left(\mathfrak{g}_{1}\right)$.

The proof for $i=2$ is similar since $\eta$ and $\xi$ are isomorphisms.

As a consequence of Lemma 3.11, we have the following characterization of $n$-Lie-isoclinism classes of Leibniz algebras.

Corollary 3.12 Given $\mathfrak{g}_{1}$ and $\mathfrak{g}_{2}$, two Leibniz algebras. Then $\mathfrak{g}_{1}$ and $\mathfrak{g}_{2}$ are $n$-Lieisoclinic if and only if there exist a Leibniz algebra $\mathfrak{h}$ that is $n$-Lie-isoclinic to $\mathfrak{g}_{1}$, and a surjective homomorphism $\theta$ from $\mathfrak{h}$ onto $\mathfrak{g}_{2}$ such that $\operatorname{Ker}(\theta) \cap \gamma_{n+1}^{\text {Lie }}(\mathfrak{h})=0$.

Proof. Assume that $\mathfrak{g}_{1}$ and $\mathfrak{g}_{2}$ are $n$-Lie-isoclinic and take $\mathfrak{h}=\mathcal{K}$. Then by the proof of Lemma 3.11, we have $\mathfrak{h} \underset{n}{\sim} \mathfrak{g}_{1}$ and the surjective homomorphism $\theta:=\tau_{2}$ : $\mathcal{K} \rightarrow \mathfrak{g}_{2}$ defined by $\tau_{2}\left(g_{1}, g_{2}\right)=g_{2}$, satisfying $\operatorname{Ker}(\theta) \cap \gamma_{n+1}^{\text {Lie }}(\mathfrak{h})=Z_{\mathfrak{g}_{1}} \cap \gamma_{n+1}^{\text {Lie }}(\mathcal{K})=0$. 
Conversely, let $\mathfrak{h}$ be a Leibniz algebra such that $\mathfrak{h} \underset{n}{\sim} \mathfrak{g}_{1}$ and $\theta: \mathfrak{h} \rightarrow \mathfrak{g}_{2}$ be a surjective homomorphism satisfying $\operatorname{Ker}(\theta) \cap \gamma_{n+1}^{\text {Lie }}(\mathfrak{h})=0$. Then by the property d) of Lemma 3.5, $\mathfrak{g}_{1} \underset{n}{\sim} \mathfrak{h} \underset{n}{\sim} \frac{\mathfrak{h}}{\operatorname{Ker}(\theta)} \cong \mathfrak{g}_{2}$.

Corollary 3.13 Given $\mathfrak{g}_{1}$ and $\mathfrak{g}_{2}$, two Leibniz algebras such that $\mathfrak{g}_{2}$ is isomorphic to $\mathfrak{g}_{1} / \mathfrak{I}$ for some two-sided ideal $\mathfrak{I}$ of $\mathfrak{g}_{1}$ satisfying $\mathfrak{I} \cap \gamma_{n+1}^{\text {Lie }}\left(\mathfrak{g}_{1}\right)=0$, then the following hold:

a) $\frac{\mathfrak{g}_{1}}{\mathcal{Z}_{n}^{\operatorname{Lie}\left(\mathfrak{g}_{1}\right)}}$ is $(n-k)$-Lie-isoclinic to $\frac{\mathfrak{g}_{2}}{\mathcal{Z}_{n}^{\text {Lie }}\left(\mathfrak{g}_{2}\right)}$, for $k=0, \ldots, n$.

b) $\gamma_{k+1}^{\mathrm{Lie}}\left(\mathfrak{g}_{1}\right)$ is $(n-k)$-Lie-isoclinic to $\gamma_{k+1}^{\mathrm{Lie}}\left(\mathfrak{g}_{2}\right)$, for $k=0, \ldots, n$.

c) $\mathfrak{g}_{1}$ is $m$-Lie-isoclinic to $\mathfrak{g}_{2}$, for all $m \geq n$.

Proof. To prove $a)$, let $\mathfrak{s}$ be a two-sided ideal of $\mathfrak{g}_{1}$ such that $\mathcal{Z}_{k}^{\text {Lie }}\left(\mathfrak{g}_{1} / \mathfrak{I}\right)=\mathfrak{s} / \mathfrak{I}$. One easily verifies that $\mathcal{Z}_{k}^{\text {Lie }}\left(\mathfrak{g}_{1}\right) \subseteq \mathfrak{s}$. Now let $g \in \mathfrak{s} \cap \gamma_{n+1-k}^{\text {Lie }}\left(\mathfrak{g}_{1}\right)$. Then for all $h_{1}, h_{2}, \ldots, h_{k} \in \mathfrak{g}_{1}$, we have $\left[\left[\left[g, h_{1}\right]_{\text {lie }}, h_{2}\right]_{\text {lie }}, \ldots, h_{k}\right]_{\text {lie }} \in \mathfrak{I} \cap \gamma_{n+1}^{\text {Lie }}\left(\mathfrak{g}_{1}\right)=0$. So $g \in \mathcal{Z}_{k}^{\text {Lie }}\left(\mathfrak{g}_{1}\right)$ and thus $\mathfrak{s} \cap \gamma_{n+1-k}^{\text {Lie }}\left(\mathfrak{g}_{1}\right) \subset \mathcal{Z}_{k}^{\text {Lie }}\left(\mathfrak{g}_{1}\right)$. This implies that $\frac{\mathfrak{s}}{\mathcal{Z}_{k}^{\text {Lie }}\left(\mathfrak{g}_{1}\right)} \cap$ $\gamma_{n+1-k}^{\text {Lie }}\left(\frac{\mathfrak{g}_{1}}{\mathcal{Z}_{k}^{\text {Lie }}\left(\mathfrak{g}_{1}\right)}\right)=0$. It follows by the property $d$ ) of Lemma 3.5 that $\frac{\mathfrak{g}_{1}}{\mathcal{Z}_{k}^{\text {Lie }}\left(\mathfrak{g}_{1}\right)}$ is $(n-k)$-Lie-isoclinic to $\frac{\mathfrak{g}_{1}}{\mathcal{Z}_{k}^{\text {Lie }}\left(\mathfrak{g}_{1}\right)} / \frac{\mathfrak{s}}{\mathcal{Z}_{k}^{\text {Lie }}\left(\mathfrak{g}_{1}\right)} \cong \mathfrak{g}_{1} / \mathfrak{s} \cong \frac{\mathfrak{g}_{1}}{\mathfrak{I}} / \frac{\mathfrak{s}}{\mathfrak{I}} \cong \frac{\mathfrak{g}_{1}}{\mathfrak{I}} / \mathcal{Z}_{n}^{\text {Lie }}\left(\frac{\mathfrak{g}_{1}}{\mathfrak{I}}\right) \cong \frac{\mathfrak{g}_{2}}{\mathcal{Z}_{k}^{\text {Lie }}\left(\mathfrak{g}_{2}\right)}$.

b) Let $k \in\{0, \ldots, n\}$ and set $\mathfrak{t}:=\mathfrak{I} \cap \gamma_{k+1}^{\text {Lie }}\left(\mathfrak{g}_{1}\right)$. It is not hard to verify that $\mathfrak{t} \cap \gamma_{n+1-k}^{\text {Lie }}\left(\mathfrak{g}_{1}\right) \subseteq \mathfrak{I} \cap \gamma_{n+1}^{\text {Lie }}\left(\mathfrak{g}_{1}\right)=0$. So again by the property $\left.d\right)$ of Lemma 3.5, we have $\gamma_{k+1}^{\text {Lie }}\left(\mathfrak{g}_{1}\right)$ is $(n-k)$-Lie-isoclinic to $\frac{\gamma_{k+1}^{\text {Lie }}\left(\mathfrak{g}_{1}\right)}{\mathfrak{t}} \cong \frac{\gamma_{k+1}^{\text {Lie }}\left(\mathfrak{g}_{1}\right)+\mathfrak{I}}{\mathfrak{I}} \cong \gamma_{k+1}^{\text {Lie }}\left(\frac{\mathfrak{g}_{1}}{\mathfrak{I}}\right) \cong \gamma_{k+1}^{\text {Lie }}\left(\mathfrak{g}_{2}\right)$.

For $c$ ), the $m$-Lie-isoclinism $\mathfrak{g}_{1} \underset{m}{\sim} \frac{\mathfrak{g}_{1}}{\mathfrak{I}} \cong \mathfrak{g}_{2}$ is obtained also by the property $d$ ) of Lemma 3.5, since $\mathfrak{I} \cap \gamma_{m+1}^{\text {Lie }}\left(\mathfrak{g}_{1}\right) \subseteq \mathfrak{I} \cap \gamma_{n+1}^{\text {Lie }}\left(\mathfrak{g}_{1}\right)=0$.

Remark 3.14 If $\mathfrak{g}_{1}$ and $\mathfrak{g}_{2}$ are $n$-Lie-isoclinic Leibniz algebras, then there exists the two-sided ideal $\mathcal{J}$ satisfying the requirements of Corollary 3.13 thanks to Corollary 3.12. Other broad class of algebras satisfying the requirements of Corollary 3.13 are Lie-nilpotent Leibniz algebras of class $n$.

Another example of non Lie-nilpotent Leibniz algebra satisfying the requirements of Corollary 3.13 is the three-dimensional Leibniz algebra $\mathfrak{g}_{1}$ with basis $\left\{a_{1}, a_{2}, a_{3}\right\}$, with bracket operation $\left[a_{1}, a_{3}\right]=a_{1}$ (see algebra $2 d$ ) in the classification given in [6] ). Take the two-sided ideal $\mathcal{J}=\left\langle\left\{a_{2}\right\}\right\rangle, \gamma_{n+1}^{\text {Lie }}\left(\mathfrak{g}_{1}\right)=\left\langle\left\{a_{1}\right\}\right\rangle$, hence the intersection is zero. Take $\mathfrak{g}_{2}=\mathfrak{g}_{1} / \mathcal{J}=\left\langle\left\{a_{1}, a_{3}\right\}\right\rangle$.

Proposition 3.15 Given $\mathfrak{g}_{1}$ and $\mathfrak{g}_{2}$, two $n$-Lie-isoclinic Leibniz algebras. Then there exist two Leibniz algebras $\mathfrak{h}_{1}$ and $\mathfrak{h}_{2}$ and a Lie-nilpotent Leibniz algebra $\mathfrak{n}$ of class at least $n$ satisfying the following:

a) $\mathfrak{g}_{1}$ is $n$-Lie-isoclinic to $\mathfrak{h}_{1} \oplus \mathfrak{n}$. 
b) $\mathfrak{g}_{2}$ is $(n-1)$-Lie-isoclinic to $\mathfrak{h}_{2}$.

c) $\mathfrak{h}_{2}$ is $n$-Lie-isoclinic to $\mathfrak{h}_{2}+\mathcal{Z}_{n}^{\text {Lie }}\left(\mathfrak{h}_{1} \oplus \mathfrak{n}\right)$, provided that $\left[Z_{n-1}^{\text {Lie }}\left(\mathfrak{g}_{2}\right), \mathfrak{g}_{2}\right]_{\text {Lie }} \subseteq$ $\gamma_{n+1}^{\mathrm{Lie}}\left(\mathfrak{g}_{2}\right)$.

d) $\mathfrak{h}_{1} \oplus \mathfrak{n}=\mathfrak{h}_{2}+\mathcal{Z}_{n}^{\text {Lie }}\left(\mathfrak{h}_{1} \oplus \mathfrak{n}\right)$ if $\mathfrak{g}_{1}$ and $\mathfrak{g}_{2}$ are finite dimensional and $\left[Z_{n-1}^{\text {Lie }}\left(\mathfrak{g}_{2}\right), \mathfrak{g}_{2}\right]_{\text {Lie }}$ $\subseteq \gamma_{n+1}^{\mathrm{Lie}}\left(\mathfrak{g}_{2}\right)$.

Proof. Keeping in mind the above notations, consider the set $\mathcal{H}=\{((g, 0)+$ $\left.\left.Z_{\mathfrak{g}_{2}},(g, 0)+\gamma_{n+1}^{\text {Lie }}(\mathcal{K})\right) / g \in \mathcal{Z}_{n}^{\text {Lie }}\left(\mathfrak{g}_{1}\right) \cap \gamma_{n}^{\text {Lie }}\left(\mathfrak{g}_{1}\right)\right\}$. It is easy to check that $\mathcal{H}$ is a two-sided ideal of $\mathcal{L}:=\mathcal{K} / Z_{\mathfrak{g}_{2}} \oplus \mathcal{K} / \gamma_{n+1}^{\text {Lie }}(\mathcal{K})$.

Now define the mappings $\alpha_{1}, \alpha_{2}: \mathcal{K} \rightarrow \mathcal{L} / \mathcal{H}$ respectively by

$$
\alpha_{1}(t)=\left(t+Z_{\mathfrak{g}_{2}}, \gamma_{n+1}^{\mathrm{Lie}}(\mathcal{K})\right)+\mathcal{H} \quad \text { and } \quad \alpha_{2}(t)=\left(t+Z_{\mathfrak{g}_{2}}, t+\gamma_{n+1}^{\mathrm{Lie}}(\mathcal{K})\right)+\mathcal{H}
$$

It is easy to check that $\alpha_{1}$ and $\alpha_{2}$ are Leibniz algebra homomorphisms with $\operatorname{Ker}\left(\alpha_{1}\right)=Z_{\mathfrak{g}_{2}}$ and $\operatorname{Ker}\left(\alpha_{2}\right)=Z_{\mathfrak{g}_{1}} \cap \gamma_{n}^{\text {Lie }}(\mathcal{K})$. Now set $\mathfrak{h}_{1}:=\alpha_{1}(\mathcal{K}), \quad \mathfrak{h}_{2}:=\alpha_{2}(\mathcal{K})$ and $\mathfrak{n}=\left\{\left(Z_{\mathfrak{g}_{2}}, t+\gamma_{n+1}^{\text {Lie }}(\mathcal{K})\right)+\mathcal{H} \mid t \in \mathcal{K}\right\}$. Then one easily verifies that $\mathfrak{n}$ is Lienilpotent of class at least $n$ since $\gamma_{j}^{\text {Lie }}(\mathfrak{n})=\left\{\left(Z_{\mathfrak{g}_{2}}, \gamma_{n+1}^{\text {Lie }}(\mathcal{K})\right)\right\}$ iff $\gamma_{j}^{\text {Lie }}(\mathcal{K}) \subseteq \gamma_{n+1}^{\text {Lie }}(\mathcal{K})$, iff $j \geq n+1$. Now combining the properties $a$ ) and $d$ ) of Lemma 3.5 and Lemma $3.11 d)$ with the first isomorphism theorem, we have

$$
\mathfrak{h}_{1} \oplus \mathfrak{n} \underset{n}{\sim} \mathfrak{h}_{1}=\alpha_{1}(\mathcal{K}) \cong \frac{\mathcal{K}}{\operatorname{Ker}\left(\alpha_{1}\right)} \cong \frac{\mathcal{K}}{Z_{\mathfrak{g}_{2}}} \cong \mathfrak{g}_{1}
$$

and

$$
\mathfrak{h}_{2}=\alpha_{2}(\mathcal{K}) \cong \frac{\mathcal{K}}{\operatorname{Ker}\left(\alpha_{2}\right)}=\frac{\mathcal{K}}{Z_{\mathfrak{g}_{1}} \cap \gamma_{n}^{\text {Lie }}(\mathcal{K})} \underset{n-1}{\sim} \frac{\mathcal{K}}{Z_{\mathfrak{g}_{1}}} \cong \mathfrak{g}_{2} .
$$

This proves $a$ ) and $b$ ).

The result $c$ ) is due to the property $b$ ) of Lemma 3.5 since $\mathfrak{h}_{2}$ is a subalgebra of $\mathfrak{h}_{1} \oplus \mathfrak{n}$ and the condition $\left[\mathcal{Z}_{n-1}^{\text {Lie }}\left(\mathfrak{h}_{2}\right), \mathfrak{h}_{2}\right]_{\text {Lie }} \subseteq \gamma_{n+1}^{\text {Lie }}\left(\mathfrak{h}_{2}\right)$ holds. Indeed, for $k=(x, y), k^{\prime}=\left(x^{\prime}, y^{\prime}\right) \in \mathcal{K}$ such that $\alpha_{1}(k) \in \mathcal{Z}_{n-1}^{\text {Lie }}\left(\mathfrak{h}_{2}\right)$, then $y \in \mathcal{Z}_{n-1}^{\text {Lie }}\left(\mathfrak{g}_{2}\right)$, since for $y_{1}, \ldots, y_{n-1} \in \mathfrak{g}_{2}$, and appropriate $x_{1}, \ldots, x_{n-1} \in \mathfrak{g}_{1}$, such that $k_{i}=$ $\left(x_{i}, y_{i}\right) \in \mathcal{K}, i=1, \ldots, n-1$, and keeping in mind that $k \in \mathcal{Z}_{n-1}^{\mathrm{Lie}}\left(\mathfrak{h}_{2}\right)$, we have $\left[\left[\left(k+Z_{\mathfrak{g}_{2}}, k+\gamma_{n+1}^{\text {Lie }}(\mathcal{K})\right),\left(k_{1}+Z_{\mathfrak{g}_{2}}, k_{1}+\gamma_{n+1}^{\text {Lie }}(\mathcal{K})\right)\right]_{l i e}, \ldots,\left(k_{n-1}+Z_{\mathfrak{g}_{2}}, k_{n-1}+\right.\right.$ $\left.\left.\gamma_{n+1}^{\text {Lie }}(\mathcal{K})\right)\right]_{l i e} \in \mathcal{H}$, i.e. $\left[\left[\left[k, k_{1}\right]_{\text {lie }}, \ldots, k_{n-1}\right]_{l i e}-(g, 0) \in Z_{\mathfrak{g}_{2}} \cap \gamma_{n+1}^{\text {Lie }}(\mathcal{K})=0\right.$ for some $g \in \mathcal{Z}_{n}^{\text {Lie }}\left(\mathfrak{g}_{1}\right) \cap \gamma_{n}^{\text {Lie }}\left(\mathfrak{g}_{1}\right)$. This implies that $\left[\left[\left[x, x_{1}\right]_{l i e}, x_{2}\right]_{l i e}, \ldots, x_{n-1}\right]_{l i e}=g$ and $\left[\left[\left[y, y_{1}\right]_{\text {lie }}, y_{2}\right]_{\text {lie }}, \ldots, y_{n-1}\right]_{\text {lie }}=0$, and thus $y \in \mathcal{Z}_{n-1}^{\mathrm{Lie}}\left(\mathfrak{g}_{2}\right)$.

Now we have $\left[\left(k+Z_{\mathfrak{g}_{2}}, k+\gamma_{n+1}^{\text {Lie }}(\mathcal{K})\right),\left(k^{\prime}+Z_{\mathfrak{g}_{2}}, k^{\prime}+\gamma_{n+1}^{\text {Lie }}(\mathcal{K})\right)\right]_{\text {lie }}+\mathcal{H}=\left(\left(\left[x, x^{\prime}\right]_{\text {lie }}\right.\right.$, $\left.\left.\left[x, x^{\prime}\right]_{\text {lie }}\right)+Z_{\mathfrak{g}_{2}},\left(\left[x, x^{\prime}\right]_{\text {lie }},\left[y, y^{\prime}\right]_{\text {lie }}\right)+\gamma_{n+1}^{\text {Lie }}(\mathcal{K})\right)+\mathcal{H} \in \gamma_{n+1}^{\text {Lie }}\left(\mathfrak{h}_{2}\right)$ since $\left[y, y^{\prime}\right]_{\text {lie }} \in$ $\left[\mathcal{Z}_{n-1}^{\mathrm{Lie}}\left(\mathfrak{g}_{2}\right), \mathfrak{g}_{2}\right]_{\text {Lie }} \subseteq \gamma_{n+1}^{\mathrm{Lie}}\left(\mathfrak{g}_{2}\right)$.

To prove $d$ ), we have by b) that $\mathfrak{g}_{2} \underset{n-1}{\sim} \mathfrak{h}_{2}$. So by the property $c$ ) of Corollary 3.13, $\mathfrak{g}_{2} \underset{n}{\sim} \mathfrak{h}_{2}$. So $\mathfrak{h}_{1} \oplus \mathfrak{n} \underset{n}{\sim} \mathfrak{g}_{1} \underset{n}{\sim} \mathfrak{g}_{2} \underset{n}{\sim} \mathfrak{h}_{2}$. The result now follows from the property $c$ ) of Lemma 3.5 since $\mathcal{K}$ is finite dimensional. Keep in mind that $\left[\mathcal{Z}_{n-1}^{\text {Lie }}\left(\mathfrak{h}_{2}\right), \mathfrak{h}_{2}\right]_{\text {Lie }} \subseteq \gamma_{n+1}^{\text {Lie }}\left(\mathfrak{h}_{2}\right)$ is showed in the proof of statement $c$ ). 
Definition 3.16 A homomorphism of $n$-Lie-central extensions $(\alpha, \beta, \gamma):\left(g_{1}\right) \rightarrow$ $\left(g_{2}\right)$ is said to be $n$-Lie-isoclinic, if there exists an isomorphism $\beta_{n+1}^{\prime}: \gamma_{n+1}^{\text {Lie }}\left(\mathfrak{g}_{1}\right) \rightarrow$ $\gamma_{n+1}^{\text {Lie }}\left(\mathfrak{g}_{2}\right)$ with $\left(\gamma, \beta_{n+1}^{\prime}\right):\left(g_{1}\right) \underset{n}{\sim}\left(g_{2}\right)$. The mapping $\beta$ is referred to as a $n$-Lieisoclinic homomorphism.

If $\beta$ is in addition an epimorphism (resp., monomorphism), then $(\alpha, \beta, \gamma)$ is called a n-Lie-isoclinic epimorphism (resp., monomorphism).

Proposition 3.17 A homomorphism of $n$-Lie-central extensions $(\alpha, \beta, \gamma):\left(g_{1}\right) \rightarrow$ $\left(g_{2}\right)$ is $n$-Lie-isoclinic if and only if $\gamma$ is an isomorphism and $\operatorname{Ker}(\beta) \cap \gamma_{n+1}^{\mathrm{Lie}}\left(\mathfrak{g}_{1}\right)=0$.

Proof. Suppose that $(\alpha, \beta, \gamma):\left(g_{1}\right) \rightarrow\left(g_{2}\right)$ is a $n$-Lie-isoclinic homomorphism. Then $\left(\gamma, \beta^{\prime}\right):\left(g_{1}\right) \underset{n}{\sim}\left(g_{2}\right)$ is a $n$-Lie-isoclinism for some isomorphism $\beta^{\prime}: \gamma_{n+1}^{\text {Lie }}\left(\mathfrak{g}_{1}\right) \rightarrow \gamma_{n+1}^{\text {Lie }}\left(\mathfrak{g}_{2}\right)$, and $\gamma: \frac{\mathfrak{g}_{1}}{\mathcal{Z}_{n}^{\text {Lie }}\left(\mathfrak{g}_{1}\right)} \rightarrow \frac{\mathfrak{g}_{2}}{\mathcal{Z}_{n}^{\text {Lie }}\left(\mathfrak{g}_{2}\right)}$ is an isomorphism. Let $g \in \operatorname{Ker}(\beta) \cap \gamma_{n+1}^{\text {Lie }}\left(\mathfrak{g}_{1}\right)$. Then $\beta(g)=0$ and $g=C_{1}^{n+1}\left(\bar{x}_{1}, \ldots, \bar{x}_{n+1}\right)$ with $\bar{x}_{i}=$ $x_{i}+\mathcal{Z}_{n}^{\text {Lie }}\left(\mathfrak{g}_{1}\right)$, for some $x_{1}, \ldots, x_{n+1} \in \mathfrak{g}_{1}$. Since $\left(\gamma, \beta^{\prime}\right):\left(g_{1}\right) \underset{n}{\sim}\left(g_{2}\right)$ is a $n$-Lieisoclinism, we have

$$
\begin{aligned}
\beta^{\prime}(g) & =\beta^{\prime}\left(C_{1}^{n+1}\left(\bar{x}_{1}, \ldots, \bar{x}_{n+1}\right)\right) \\
& =C_{2}^{n+1}\left(\gamma^{n+1}\left(\bar{x}_{1}, \ldots, \bar{x}_{n+1}\right)\right) \\
& =C_{2}^{n+1}\left(\gamma\left(\bar{x}_{1}\right), \ldots, \gamma\left(\widetilde{x_{n+1}}\right)\right) \\
& =C_{2}^{n+1}\left(\widetilde{\beta\left(x_{1}\right)}, \ldots, \widehat{\beta\left(x_{n+1}\right)}\right) \\
& =\beta\left(C_{1}^{n+1}\left(\bar{x}_{1}, \ldots, \bar{x}_{n+1}\right)\right)=\beta(g)=0 .
\end{aligned}
$$

Therefore $g=0$ since $\beta^{\prime}$ is one-to-one.

Conversely, suppose that $\operatorname{Ker}(\beta) \cap \gamma_{n+1}^{\text {Lie }}\left(\mathfrak{g}_{1}\right)=0$, then define $\beta^{\prime}: \gamma_{n+1}^{\text {Lie }}\left(\mathfrak{g}_{1}\right) \rightarrow$ $\gamma_{n+1}^{\text {Lie }}\left(\mathfrak{g}_{2}\right)$ by $\beta^{\prime}(g)=\beta(g)$. Clearly $\beta^{\prime}$ is one-to-one due to $\operatorname{Ker}(\beta) \cap \gamma_{n+1}^{\text {Lie }}\left(\mathfrak{g}_{1}\right)=0$. To show that $\beta^{\prime}$ is onto, let $h \in \gamma_{n}^{\text {Lie }}\left(\mathfrak{g}_{2}\right)$. Then $h=\left[\left[\left[y_{1}, y_{2}\right]_{l i e}, y_{3}\right]_{l i e}, \ldots, y_{n+1}\right]_{l i e}=$ $C_{2}^{n+1}\left(\tilde{y}_{1}, \ldots, \tilde{y}_{n+1}\right)$ for some $y_{1}, \ldots, y_{n+1} \in \mathfrak{g}_{2}$. Since $\gamma_{1}$ is onto, we have $\tilde{y}_{i}=$ $\gamma\left(\bar{x}_{i}\right)=\widetilde{\beta\left(x_{i}\right)}, \quad i=1, \ldots, n+1$. It follows that

$$
\begin{aligned}
\beta^{\prime}\left(C_{1}^{n+1}\left(\bar{x}_{1}, \ldots, \bar{x}_{n+1}\right)\right) & =\beta\left(C_{1}^{n+1}\left(\bar{x}_{1}, \ldots, \bar{x}_{n+1}\right)\right) \\
& =C_{2}^{n+1}\left(\widetilde{\beta\left(x_{1}\right)}, \ldots, \widehat{\beta\left(x_{n+1}\right)}\right) \\
& =C_{2}^{n+1}\left(\tilde{y}_{1}, \ldots, \tilde{y}_{n+1}\right)=y .
\end{aligned}
$$

Remark 3.18 From the proof of Proposition 3.17, it follows that the isomorphism $\beta_{n+1}^{\prime}$ in Definition 3.16 is the restriction of $\beta$ to $\gamma_{n+1}^{\mathrm{Lie}}\left(\mathfrak{g}_{1}\right)$.

Proposition 3.19 Let $\beta: \mathfrak{g}_{1} \rightarrow \mathfrak{g}_{2}$ be a homomorphism of Leibniz algebras. Then $\beta$ induces a $n$-Lie-isoclinic homomorphism from $\left(g_{1}\right)$ to $\left(g_{2}\right)$ if and only if $\operatorname{Ker}(\beta) \cap \gamma_{n+1}^{\text {Lie }}\left(\mathfrak{g}_{1}\right)=0$ and $\operatorname{Im}(\beta)+\mathcal{Z}_{n}^{\text {Lie }}\left(\mathfrak{g}_{2}\right)=\mathfrak{g}_{2}$. 
Proof. Let $(\alpha, \beta, \gamma):\left(g_{1}\right) \rightarrow\left(g_{2}\right)$ be a $n$-Lie-isoclinic homomorphism induced by $\beta$. Then by Proposition 3.17, $\operatorname{Ker}(\beta) \cap \gamma_{n+1}^{\text {Lie }}\left(\mathfrak{g}_{1}\right)=0$. To show that $\operatorname{Im}(\beta)+$ $\mathcal{Z}_{n}^{\text {Lie }}\left(\mathfrak{g}_{2}\right)=\mathfrak{g}_{2}$, let $h \in \mathfrak{g}_{2}$. Since $\gamma$ is onto and $(\alpha, \beta, \gamma)$ is a homomorphism of $n$-Lie-central extensions, we have $\tilde{h}=\gamma(\bar{g})=\widehat{\beta(g)}$ for some $g \in \mathfrak{g}_{1}$. It follows that $h-\beta(g) \in \mathcal{Z}_{\text {Lie }}\left(\mathfrak{g}_{2}\right)$ i.e, $h=\beta(g)+z$ for some $z \in \mathcal{Z}_{\text {Lie }}\left(\mathfrak{g}_{2}\right)$. This proves $\mathfrak{g}_{2} \subseteq \operatorname{Im}(\beta)+\mathcal{Z}_{n}^{\text {Lie }}\left(\mathfrak{g}_{2}\right)$, the other inclusion being obvious.

Conversely, suppose that $\operatorname{Ker}(\beta) \cap \gamma_{n+1}^{\text {Lie }}\left(\mathfrak{g}_{1}\right)=0$ and $\operatorname{Im}(\beta)+\mathcal{Z}_{n}^{\text {Lie }}\left(\mathfrak{g}_{2}\right)=\mathfrak{g}_{2}$. We claim that the maps $\alpha:=\left.\beta\right|_{\mathcal{Z}_{n}^{\text {Lie }\left(\mathfrak{g}_{1}\right)}}$ and $\gamma: \mathfrak{g}_{1} / \mathcal{Z}_{n}^{\text {Lie }}\left(\mathfrak{g}_{1}\right) \rightarrow \mathfrak{g}_{2} / \mathcal{Z}_{n}^{\text {Lie }}\left(\mathfrak{g}_{2}\right)$ defined by $\gamma(\bar{g})=\widetilde{\beta(g)}$ are well-defined homomorphisms for every integer $n$. We prove by induction that $\beta\left(\mathcal{Z}_{n}^{\text {Lie }}\left(\mathfrak{g}_{1}\right)\right) \subseteq \mathcal{Z}_{n}^{\text {Lie }}\left(\mathfrak{g}_{2}\right)$. Let $x \in \mathcal{Z}_{n}^{\text {Lie }}\left(\mathfrak{g}_{1}\right)$ and $h \in \mathfrak{g}_{2}$, then $h=\beta(g)+z$ for some $g \in \mathfrak{g}_{1}$ and $z \in \mathcal{Z}_{n}^{\text {Lie }}\left(\mathfrak{g}_{2}\right)$. By inductive hypothesis, we have $\beta\left([x, g]_{\text {lie }}\right) \in \mathcal{Z}_{n-1}^{\text {Lie }}\left(\mathfrak{g}_{2}\right)$ as $[x, g]_{\text {lie }} \in \mathcal{Z}_{n-1}^{\text {Lie }}\left(\mathfrak{g}_{1}\right)$, so

$$
\begin{aligned}
{[\beta(x), h]_{l i e} } & =[\beta(x), \beta(g)+z]_{\text {lie }} \\
& =[\beta(x), \beta(g)]_{\text {lie }}+[\beta(x), z]_{\text {lie }} \\
& =\beta\left([x, g]_{\text {lie }}\right)+[\beta(x), z]_{\text {lie }} \in \mathcal{Z}_{n-1}^{\mathrm{Lie}}\left(\mathfrak{g}_{2}\right),
\end{aligned}
$$

and thus $\beta(x) \in \mathcal{Z}_{n}^{\text {Lie }}\left(\mathfrak{g}_{2}\right)$. One easily verifies that $(\alpha, \beta, \gamma):\left(g_{1}\right) \rightarrow\left(g_{2}\right)$ is a homomorphism of $n$-Lie-central extensions. It remains to show that it is $n$-Lie-isoclinic. By Proposition 3.17, it is enough to show that $\gamma$ is an isomorphism. $\gamma$ is onto because every $h \in \mathfrak{g}_{2}$ can be written as $h=\beta(g)+z$ for some $g \in \mathfrak{g}_{1}$ and $z \in \mathcal{Z}_{n}^{\text {Lie }}\left(\mathfrak{g}_{2}\right)$, yielding $\bar{h}=\widetilde{\beta(g)}=\gamma(\bar{g})$. To show that $\gamma$ is one-to-one, let $x \in \mathfrak{g}_{1}$ satisfying $\gamma(\bar{x})=0$, i.e. $\beta(x) \in \mathcal{Z}_{n}^{\text {Lie }}\left(\mathfrak{g}_{2}\right)$. Let $g_{1}, \ldots, g_{n} \in \mathfrak{g}_{1}$. We need to show that $t:=\left[\left[\left[x, g_{1}\right]_{l i e}, g_{2}\right]_{l i e}, \ldots, g_{n}\right]_{l i e}=0$. Indeed, $\beta(t)=\left[\left[\left[\beta(x), \beta\left(g_{1}\right)\right]_{l i e}, \beta\left(g_{2}\right)\right]_{l i e}, \ldots, \beta\left(g_{n}\right)\right]_{l i e}=0$ since $\beta(x) \in \mathcal{Z}_{n}^{\text {Lie }}\left(\mathfrak{g}_{2}\right)$. So $t \in \operatorname{Ker}(\beta) \cap \gamma_{n+1}^{\text {Lie }}\left(\mathfrak{g}_{1}\right)=0$. Hence $x \in \mathcal{Z}_{n}^{\text {Lie }}\left(\mathfrak{g}_{1}\right)$. This completes the proof.

\section{Some properties on $n$-Lie-stem Leibniz alge- bras}

Definition 4.1 A Leibniz algebra $\mathfrak{g}$ is said to be $n$-Lie-stem Leibniz algebra when$\operatorname{ever} \mathcal{Z}_{n}^{\text {Lie }}(\mathfrak{g}) \subseteq \gamma_{n+1}^{\text {Lie }}(\mathfrak{g})$.

The lemma below characterizes $n$-Lie-stem Leibniz algebras.

Lemma 4.2 A Leibniz algebra $\mathfrak{g}$ is a $n$-Lie-stem Leibniz algebra if and only if $\mathfrak{I} \cap \gamma_{n+1}^{\text {Lie }}(\mathfrak{g}) \neq 0$ for all nonzero two-sided ideal $\mathfrak{I}$ of $\mathfrak{g}$.

Proof. Assume that $\mathfrak{g}$ is not a $n$-Lie-stem Leibniz algebra and let $g \in \mathcal{Z}_{n}^{\text {Lie }}(\mathfrak{g})$ with $g \notin \gamma_{n+1}^{\text {Lie }}(\mathfrak{g})$. Consider the two-sided ideal $\mathfrak{I}:=\operatorname{span}\{g\}$. Then $\mathfrak{I}$ is a nonzero two-sided ideal of $\mathfrak{g}$ satisfying $\mathfrak{I} \cap \gamma_{n+1}^{\text {Lie }}(\mathfrak{g})=0$. 
Conversely, suppose that $\mathfrak{g}$ is a $n$-Lie-stem Leibniz algebra and let $\mathfrak{I}$ be a two-sided ideal of $\mathfrak{g}$ satisfying $\mathfrak{I} \cap \gamma_{n+1}^{\text {Lie }}(\mathfrak{g})=0$. Then $\mathfrak{I} \subseteq \mathcal{Z}_{n}^{\text {Lie }}(\mathfrak{g})$ since $\left[\left[\left[x, g_{1}\right]_{\text {lie }}, g_{2}\right]_{\text {lie }}, \ldots, g_{n+1}\right]_{\text {lie }} \in \mathfrak{I} \cap \gamma_{n+1}^{\text {Lie }}(\mathfrak{g})=0$ for all $x \in \mathfrak{I}$ and $g_{1}, \ldots, g_{n+1} \in \mathfrak{g}$. So $\mathfrak{I}=\mathfrak{I} \cap \mathcal{Z}_{n}^{\text {Lie }}(\mathfrak{g}) \subseteq \mathfrak{I} \cap \gamma_{n+1}^{\text {Lie }}(\mathfrak{g})=0$, which implies that $\mathfrak{I}=0$.

Corollary 4.3 Every Leibniz algebra is n-Lie-isoclinic to some $n$-Lie-stem Leibniz algebra.

Proof. Consider the set $\mathfrak{M}=\left\{\mathfrak{I} \mid\right.$ two-sided ideal of $\mathfrak{g}$ satisfying $\mathfrak{I} \cap \gamma_{n+1}^{\text {Lie }}(\mathfrak{g})=$ $0\}$. This set contains the ideal $\mathfrak{I}=0$. So $\mathfrak{M}$ is non-empty and partially ordered by set inclusion. By Zorn's lemma, it contains a maximal two-sided ideal, call it $\mathfrak{m}$. By the property $d$ ) of Lemma 3.5, it follows that $\mathfrak{g}$ is $n$-Lie-isoclinic to $\mathfrak{g} / \mathfrak{m}$ since $\mathfrak{m} \cap \gamma_{n+1}^{\text {Lie }}(\mathfrak{g})=0$. It remains to show that $\mathfrak{g} / \mathfrak{m}$ is a $n$-Lie-stem Leibniz algebra. Let $\mathfrak{I}$ be an arbitrary two-sided ideal of $\mathfrak{g}$ containing $\mathfrak{m}$ and satisfying $\frac{\mathfrak{I}}{\mathfrak{m}} \cap \gamma_{n+1}^{\text {Lie }}\left(\frac{\mathfrak{g}}{\mathfrak{m}}\right)=\mathfrak{m}$. By Lemma 4.2, it is enough to show that $\mathfrak{I} \subseteq \mathfrak{m}$. First, we prove that $\mathfrak{I} \cap \gamma_{n+1}^{\text {Lie }}(\mathfrak{g})=0$. Let $x \in \mathfrak{I} \cap \gamma_{n+1}^{\text {Lie }}(\mathfrak{g})$, then $x+\mathfrak{m} \in \frac{\mathfrak{I}}{\mathfrak{m}} \cap \frac{\gamma_{n+1}^{\text {Lie }}(\mathfrak{g})}{\mathfrak{m}}=$ $\frac{\mathfrak{I}}{\mathfrak{m}} \cap \gamma_{n+1}^{\text {Lie }}\left(\frac{\mathfrak{g}}{\mathfrak{m}}\right)=\mathfrak{m}$. So $x \in \mathfrak{m}$ and thus $x \in \mathfrak{m} \cap \gamma_{n+1}^{\text {Lie }}(\mathfrak{g})=0$. Hence $\mathfrak{I} \cap \gamma_{n+1}^{\text {Lie }}(\mathfrak{g})=0$. This implies that $\mathfrak{I} \in \mathfrak{M}$, therefore $\mathfrak{I} \subseteq \mathfrak{m}$ by maximality of $\mathfrak{m}$.

Recall from [1, Definition 5.4] that the Frattini subalgebra of an algebra $\mathfrak{g}$ is $\Phi(\mathfrak{g})=\bigcap_{\mathfrak{m} \in \mathfrak{S}} \mathfrak{m}$, where $\mathfrak{S}$ is the set of all maximal subalgebras of $\mathfrak{g}$.

The following classifies $n$-Lie-stem Leibniz algebras with trivial Frattini subalgebras.

Proposition 4.4 Let $\mathfrak{g}$ be a Leibniz algebra. If the Frattini subalgebra of $\mathfrak{g}$ is trivial, then up to isomorphism, there is only one $n$-Lie-stem Leibniz algebra that is $n$-Lie-isoclinic to $\mathfrak{g}$. This $n$-Lie-stem Leibniz algebra is precisely $\frac{\mathfrak{g}}{\mathcal{Z}_{n}^{\text {Lie }}(\mathfrak{g})}$.

Proof. Let $\mathfrak{s}$ be a $n$-Lie-stem Leibniz algebra that is $n$-Lie-isoclinic to $\mathfrak{g}$. Then by definition, $\frac{\mathfrak{s}}{\mathcal{Z}_{n}^{\text {Lie }(\mathfrak{s})}} \stackrel{\xi}{\cong} \frac{\mathfrak{g}}{\mathcal{Z}_{n}^{\text {Lie }}(\mathfrak{g})}$. So it is enough to prove that $\mathcal{Z}_{n}^{\text {Lie }}(\mathfrak{s})=0$. Let $\mathfrak{m}$ be a maximal subalgebra of $\mathfrak{g}$. Then either $\mathcal{Z}_{n}^{\text {Lie }}(\mathfrak{g}) \subseteq \mathfrak{m}$ or $\mathcal{Z}_{n}^{\text {Lie }}(\mathfrak{g})+\mathfrak{m}=\mathfrak{g}$. Since $\gamma_{n+1}^{\text {Lie }}\left(\mathcal{Z}_{n}^{\text {Lie }}(\mathfrak{g})\right)=0$, it follows that $\mathcal{Z}_{n}^{\text {Lie }}(\mathfrak{g}) \cap \gamma_{n+1}^{\text {Lie }}(\mathfrak{g}) \subseteq \mathfrak{m}+\gamma_{n+1}^{\text {Lie }}(\mathfrak{m})=\mathfrak{m}$. So by the property b) of Proposition 3.10 , we have $\xi\left(\mathcal{Z}_{n}^{\text {Lie }}(\mathfrak{s}) \cap \gamma_{n+1}^{\text {Lie }}(\mathfrak{s})\right)=\mathcal{Z}_{n}^{\text {Lie }}(\mathfrak{g}) \cap$ $\gamma_{n+1}^{\text {Lie }}(\mathfrak{g}) \subseteq \bigcap_{\mathfrak{m} \in \mathfrak{S}} \mathfrak{m}=\Phi(\mathfrak{g})=0$. Thus $\mathcal{Z}_{n}^{\text {Lie }}(\mathfrak{s}) \cap \gamma_{n+1}^{\text {Lie }}(\mathfrak{s})=0$ as $\xi$ is an isomorphism. Therefore $\mathcal{Z}_{n}^{\text {Lie }}(\mathfrak{s})=0$ by Lemma 4.2 since $\mathfrak{s}$ is a $n$-Lie-stem Leibniz algebra .

Theorem 4.5 Let $\mathfrak{g}$ be a Leibniz algebra. A finite-dimensional Leibniz algebra $\mathfrak{q}$, such that $\mathfrak{q} \underset{n}{\sim} \mathfrak{g}$, is a n-Lie-stem Leibniz algebra if and only if $\operatorname{dim}(\mathfrak{q})=$ $\min \{\operatorname{dim}(\mathfrak{h}) \mid \mathfrak{h} \underset{n}{\sim} \mathfrak{g}\}$. 
Proof. Let $\mathfrak{q} \underset{n}{\sim} \mathfrak{g}$ and $\mathfrak{h} \underset{n}{\sim} \mathfrak{g}$ be and assume that $\mathfrak{q}$ is a finite-dimensional $n$-Lie-stem Leibniz algebra. Then we have

$$
\begin{aligned}
& \frac{\gamma_{n+1}^{\text {Lie }}(\mathfrak{h})}{\gamma_{n+1}^{\text {Lie }}(\mathfrak{h}) \cap \mathcal{Z}_{n}^{\text {Lie }}(\mathfrak{h})} \cong \frac{\gamma_{n+1}^{\text {Lie }}(\mathfrak{h})+\mathcal{Z}_{n}^{\text {Lie }}(\mathfrak{h})}{\mathcal{Z}_{n}^{\text {Lie }}(\mathfrak{h})} \\
& \cong \frac{\gamma_{n+1}^{\text {Lie }}(\mathfrak{h})}{\mathcal{Z}_{n}^{\text {Lie }(\mathfrak{h})}} \\
& \cong \gamma_{n+1}^{\text {Lie }}\left(\frac{\mathfrak{h}}{\mathcal{Z}_{n}^{\text {Lie }(\mathfrak{h})}}\right) \\
& \cong \gamma_{n+1}^{\text {Lie }}\left(\frac{\mathfrak{q}}{\mathcal{Z}_{n}^{\text {Lie }(\mathfrak{q})}}\right) \\
& \cong \frac{\gamma_{n+1}^{\mathrm{Lie}}(\mathfrak{q})}{\mathcal{Z}_{n}^{\mathrm{Lie}}(\mathfrak{q})}
\end{aligned}
$$

and $\gamma_{n+1}^{\text {Lie }}(\mathfrak{q}) \cong \gamma_{n+1}^{\text {Lie }}(\mathfrak{h})$. So $\operatorname{dim}\left(\mathcal{Z}_{n}^{\text {Lie }}(\mathfrak{q})\right)=\operatorname{dim}\left(\gamma_{n+1}^{\text {Lie }}(\mathfrak{h}) \cap \mathcal{Z}_{n}^{\text {Lie }}(\mathfrak{h})\right) \leq \operatorname{dim}\left(\mathcal{Z}_{n}^{\text {Lie }}(\mathfrak{h})\right)$. On the other hand $\frac{\mathfrak{h}}{\mathcal{Z}_{n}^{\operatorname{Lie}(\mathfrak{h})}} \cong \frac{\mathfrak{q}}{\mathcal{Z}_{n}^{\text {Lie }}(\mathfrak{q})}$. Therefore $\operatorname{dim}(\mathfrak{q}) \leq \operatorname{dim}(\mathfrak{h})$.

Conversely, let $\mathfrak{q} \underset{n}{\sim} \mathfrak{g}$ such that $\mathfrak{q}$ has the minimum dimension. Owing to Corollary 4.3 there is a two-sided ideal $\mathfrak{h}$ of $\mathfrak{q}$ contained in $\mathcal{Z}_{n}^{\text {Lie }}(\mathfrak{q})$ such that $\mathfrak{q} \underset{n}{\sim} \mathfrak{\mathfrak { h }}$ and $\mathcal{Z}_{n}^{\text {Lie }}(\mathfrak{q})=\left(\gamma_{n+1}^{\text {Lie }}(\mathfrak{q}) \cap \mathcal{Z}_{n}^{\text {Lie }}(\mathfrak{q})\right) \oplus \mathfrak{h}$. But $\mathfrak{q}$ has minimum dimension, which implies that $\mathfrak{h}=0$, therefore $\mathcal{Z}_{n}^{\text {Lie }}(\mathfrak{q}) \subseteq \gamma_{n+1}^{\text {Lie }}(\mathfrak{q})$ and this completes the proof.

Theorem 4.6 If $\mathfrak{g}$ and $\mathfrak{q}$ are two $n$-Lie-isoclinic $n$-Lie-stem Leibniz algebras then $\mathcal{Z}_{n}^{\text {Lie }}(\mathfrak{g}) \cong \mathcal{Z}_{n}^{\text {Lie }}(\mathfrak{q})$.

Proof. Let $\mathfrak{g}$ and $\mathfrak{q}$ be two $n$-Lie-isoclinic $n$-Lie-stem Leibniz algebras. In view of proof of Theorem 4.5 and isomorphism $\xi: \gamma_{n+1}^{\text {Lie }}(\mathfrak{g}) \longrightarrow \gamma_{n+1}^{\mathrm{Lie}}(\mathfrak{q})$ we have the following commutative diagram with exact rows:

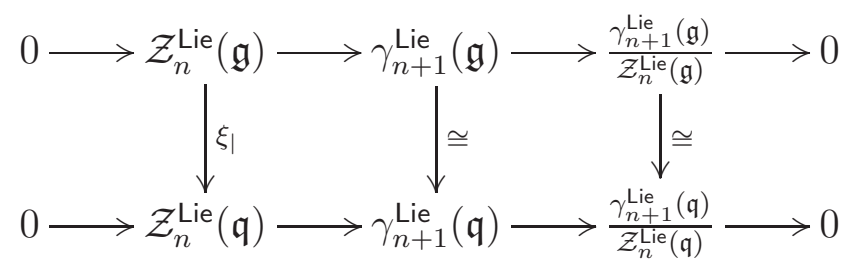

where $\xi\left(\mathcal{Z}_{n}^{\text {Lie }}(\mathfrak{g})\right) \subseteq \mathcal{Z}_{n}^{\text {Lie }}(\mathfrak{q})$ since for all $x \in \gamma_{n+1}^{\text {Lie }}(\mathfrak{g}), \eta\left(x+\mathcal{Z}_{n}^{\text {Lie }}(\mathfrak{g})\right)=\xi(x)+$ $\mathcal{Z}_{n}^{\text {Lie }}(\mathfrak{q})$. Hence, for $x \in \mathcal{Z}_{n}^{\text {Lie }}(\mathfrak{g}), 0=\eta\left(\pi_{\mathfrak{g}}(x)\right)=\xi(x)+\mathcal{Z}_{n}^{\text {Lie }}(\mathfrak{q})$ (here $\pi_{\mathfrak{g}}$ : $\mathfrak{g} \rightarrow \mathfrak{g} / \mathcal{Z}_{n}^{\text {Lie }}(\mathfrak{g})$ denotes the canonical projection), then $\xi(x) \in \mathcal{Z}_{n}^{\text {Lie }}(\mathfrak{q})$. Now the Snake Lemma yields $\xi$ is a surjective homomorphism and so $\xi\left(\mathcal{Z}_{n}^{\text {Lie }}(\mathfrak{g})\right)=\mathcal{Z}_{n}^{\text {Lie }}(\mathfrak{q})$. Moreover the left hand square is a pull-back diagram, then $\xi_{\mid}$is an injective homomorphism. Hence the isomorphism $\mathcal{Z}_{n}^{\text {Lie }}(\mathfrak{g}) \cong \mathcal{Z}_{n}^{\text {Lie }}(\mathfrak{q})$. 


\section{Acknowledgements}

Second author was supported by Agencia Estatal de Investigación (Spain), grant

MTM2016-79661-P (AEI/FEDER, UE, support included).

\section{References}

[1] D. W. Barnes: Some theorems on Leibniz algebras, Comm. Algebra 39 (7) (2011), 2463-2472.

[2] J. C. Bioch: On n-isoclinic groups, Indag. Math. 38 (1976), 400-407.

[3] G. R. Biyogmam and J. M. Casas: On Lie-isoclinic Leibniz algebras, J. Algebra 499 (2018), 337-357. DOI: 10.1016/j.jalgebra.2017.01.034.

[4] G. R. Biyogmam and J. M. Casas: The c-nilpotent Schur Lie-multiplier of Leibniz algebras, arXiv: 1717:10173 (2017).

[5] E. M. Cañete and A. Kh. Khudoyberdiyev: The classification of 4-dimensional Leibniz algebras, Linear Algebra Appl. 439 (1) (2013), 273-288.

[6] J. M. Casas, M. A. Insua, M. Ladra and S. Ladra: An algorithm for the classification of 3-dimensional complex Leibniz algebras, Linear Algebra Appl. 436 (9) (2012), 3747-3756.

[7] J. M. Casas and E. Khmaladze: On Lie-central extensions of Leibniz algebras, Rev. R. Acad. Cienc. Exactas Fís. Nat. Ser. A Math. RACSAM 111 (1) (2017). 36-56. DOI 10.1007/s13398-016-0274-6.

[8] J. M. Casas and T. Van der Linden: Universal central extensions in semiabelian categories, Appl. Categor. Struct. 22 (1) (2014), 253-268.

[9] A. Erfanian, R. Rezaei and F. G. Russo: Relative n-isoclinism classes and relative $n$-th nilpotency degree of finite groups, Filomat 27 (2) (2013), 365369.

[10] P. Hall: The classification of prime-power groups, J. Reine Angew. Math. 182 (1940), 130-141.

[11] P. Hall: Verbal and marginal subgroups, J. Reine Angew. Math. 182 (1940), $156-157$.

[12] S. Heidarian and A. Gholami: On n-isoclinic pairs of groups, Algebra Colloq. 18 (1) (2011), 999-1006.

[13] N. S. Hekster: On the Structure of $n$-isoclinism classes of groups, J. Pure Appl. Algebra 40 (1986), 63-85. 
[14] J.-L. Loday: Cyclic homology, Grundl. Math. Wiss. Bd. 301, Springer, Berlin (1992).

[15] J.-L. Loday: Une version non commutative des algèbres de Lie: les algèbres de Leibniz, L'Enseignement Mathématique 39 (1993), 269-292.

[16] M. R. R. Moghaddam, F. Saeedi, S. Tajnia and B. Veisi: Characterization of relative n-isoclinism of a pair of Lie algebras, Quaest. Math. 38 (1) (2015), $27-39$.

[17] M. R. Moghaddam, A. R. Salemkar and K. Chiti: n-isoclinism classes and n-nilpotency degree of finite groups, Algebra Colloq. 12 (2) (2005), 255-261.

[18] F. Parvaneh, R. R. Mohammad and A. Khaksar: Some properties of nisoclinism in Lie algebras, Ital. J. Pure Appl. Math. 28 (2011), 167-178.

[19] A. R. Salemkar, F. Mirzaei: Characterizing n-isoclinism classes of Lie algebras, Comm. Algebra 38 (9) (2010), 3392-3403.

[20] R. W. van der Waall: On n-isoclinic embedding of n-isoclinic groups, Indag. Math. 15 (4) (2004), 595-600. 